\title{
The Opioid System and Food Intake: Use of Opiate Antagonists in Treatment of Binge Eating Disorder and Abnormal Eating Behavior
}

\author{
Leon P. Valbrun ${ }^{a}$, Valeriy Zvonarev ${ }^{\mathrm{b}, \mathrm{c}}$
}

\begin{abstract}
Eating disorders (EDs) and substance use disorders (SUDs) commonly co-occur, especially in conjunction with affective syndromes, yet little is known about opiate abuse and ED symptoms in patients on naltrexone-bupropion therapy. Moreover, evidence suggests that the opioid system can also be regarded as one of the major systems regulating the anticipatory processes preceding binge eating episodes. The lack of evidence in the effectiveness of psychotherapy treatment in addition to psychotropic mediations compounds the difficulties in stabilizing individuals with EDs. This article aims to exhaustively review literature relating to the use of opioid antagonists in the management of binge eating disorder (BED) and other abnormal eating habits and how this can be augmented by the use of psychological approaches to come up with the most effective therapy or combination of therapies to manage these conditions. Although this approach is promising, it has not been evaluated. A review of the literature pertaining to the use of naltrexone in patients with EDs was performed through PubMed, PsycINFO and MEDLINE. We selected 63 relevant articles published between 1981 and 2018 and those written in English. Search terms included "Opioid antagonists", "naltrexone", "bupropion" and "Psychotherapy" each combined with "Binge Eating Disorder", "Bulimia Nervosa", "Anorexia Nervosa", "Eating Disorder", "EDNOS" and "Obesity". While working with these articles, we also identified several problems related to use of these methods in real clinical practice. Seventy-seven articles were reviewed, and 63 were selected for inclusion. Data obtained from these sources confirmed that the blockade of opioid receptors diminishes food intake. More recent findings also indicate that the combination of bupropion and naltrexone can induce weight loss. Augmentation of this by introducing psychotherapy may lead to better outcomes. Cognitive behavioral therapy (CBT) was the most frequently recommended psychotherapy intervention, showing efficacy for EDs and chemical addictions as
\end{abstract}

Manuscript submitted December 11, 2019, accepted December 28, 2019

aDepartment of Psychiatry, Interfaith Medical Center, 1545 Atlantic Avenue, Brooklyn, NY 11213, USA

bSchool of Behavioral Sciences, California Southern University, 3330 Harbor Blvd, Costa Mesa, CA 92626, USA

${ }^{\mathrm{c} C o r r e s p o n d i n g ~ A u t h o r: ~ V a l e r i y ~ Z v o n a r e v, ~ S c h o o l ~ o f ~ B e h a v i o r a l ~ S c i e n c e s, ~}$ California Southern University, 3330 Harbor Blvd, Costa Mesa, CA 92626, USA. Email: zvonarevval@gmail.com

doi: https://doi.org/10.14740/jocmr4066 documented by most of the studies, but with uncertain efficacy when utilized as augmentation strategy. There are limited data supporting the use of psychotherapy in augmentation of standard therapy in ED; however, there is evidence to support that psychotherapy is safe in this population and has been effective in cases of patients with opiate addiction with and without psychiatric comorbidities as well as BED. More research is needed to establish treatment guidelines. Combining pharmacotherapeutic and psychotherapeutic interventions leads to the achievement of a better outcome in managing patients with EDs. Involving families or the use of support groups increases chances of adherence to the prescribed interventions resulting in higher rates of remission. However, it is clear that all of these interventions must occur in the context of a comprehensive treatment program. We believe that patient-specific psychotherapy may not only facilitate the treatment process, but also cause significant alterations in eating pattern. This approach for BED may lead to more significant treatment outcomes, but this possibility must be tested in larger samples.

Keywords: Opiates; Binge eating; Naltrexone; Psychotherapy

\section{Introduction}

Many studies have highlighted the parallels between binge eating behavior and substance abuse. It is clear that some aspects of binge eating could meet the Diagnostic and Statistical Manual of Mental Disorders (DSM) diagnostic criteria for substance abuse. Moreover, several studies have discussed the possibility that opioid system dysregulation could underlie addictive binge eating. There is considerable evidence that antagonism of the endogenous opioids will suppress food intake in a variety of animal species [1]. Interestingly, some studies in bulimic patients treated with opioid receptor antagonists showed a reduction in the frequency of binging following naltrexone administration, and improvements in most patients' bingerelated indices [1]. This included both the number of binges and purges as well as the ratio of binge to normal eating. In obesity and eating disorders (EDs), food craving is a form of anticipatory reward that is regulated by endogenous opioid and mesolimbic dopaminergic systems. Naltrexone non-specific blockade of the opioid receptors blocks the endogenous opioid (i.e. endorphinic) action. Additionally, cells of the hypothalamic proopiomelanocortin (POMC) play an important role in 
regulating appetite, as they send a stop-food signal to the brain secreting the melanocyte-stimulating hormone (MSH). Since naltrexone blocks feedback inhibition, it could provide continuous release of $\mathrm{MSH}$, which leads to a decrease in appetite.

Psychotherapy can be thought of as a specific type of treatment protocol that promotes social and emotional development, neural integration and processing complexity in patients with EDs. A number of studies on brain changes after psychotherapy for depression, anxiety disorders and borderline personality disorder (BPD) have been published. In one of them, the researchers compared behavior therapy with fluoxetine treatment. Both treatment modalities demonstrated similar changes in the brain, especially in the caudate nucleus. These studies suggest that psychotherapy alters brain function in patients suffering from major psychiatric disorders. However, no one has discussed these changes in ED patients.

In this paper, we provide a brief overview of the opioid system, and review the rationales and data supporting the effectiveness of specific medications in treating patients with EDs with the primary focus on binge eating disorder (BED). We conclude by summarizing these data, discussing the role of psychotherapy as an augmentation strategy and suggesting future areas for research.

\section{Role of the Opioid System in Food Intake: Ho- meostatic and Hedonic Mechanisms}

Eating behavior is controlled by a complicated system, which includes the central and humoral regulating elements. The key role in ensuring the central mechanisms functioning is played by the cortex and reward zones in the limbic system (hedonic regulation), where the stimuli originating from the environment are analyzed; they include both alimentary (appearance, taste and food smell) and non-alimentary stimuli (emotional discomfort and stress). Stimulation of the ventromedial nuclei of hypothalamus is accompanied by a decrease in appetite, while stimulation of the lateral nuclei is accompanied by its augmentation [1]. Opioids act as neurotransmitters when released by arcuate nucleus neurons projected onto both the ventral tegmental area (VTA) and the nucleus accumbens (NAcc). Endogenous opioid peptides include endorphins, enkephalins, dynorphins and endomorphins and act through three types of receptors: $\mu-, \delta$ - and $\kappa$-opioid receptors (MOR, DOR and KOR), which belong to the $G$ protein-coupled receptor superfamily (Fig. 1).

Most opiate-dependent metabolites are produced from common precursor proteins, pro-opiomelanocortin $(\alpha-\mathrm{MSH}$, $\gamma$-MSH, $\beta$-MSH, adrenocorticotropic hormone (ACTH), $\beta$-endorphin, $\alpha$-endorphin, $\gamma$-endorphin, $\beta$-lipoprotein $(\beta-\mathrm{LPH}), \gamma-\mathrm{LPH}$, corticotropin-like intermediate lobe peptide (CLIP)), prodynorphin (dynorphin A, dynorphin B, $\alpha$-neoendorphin, $\beta$-neo-endorphin, dynorphin-32, leu-morphine), preproenkephalin (leu-enkephalin, met-enkephalin, amidorphin, adrenorphin, peptide B, peptide E, peptide F), preproneu-ropeptide FF (NPFF), etc. Parts of these precursor proteins are cleaved producing endorphins, enkephalins or dynorphins, stored in opioid neurons, and presumably released during neu- rotransmission in order to mediate the action of endogenous opioids including their role in reinforcement and satisfaction in the reinforcement scheme. $\beta$-endorphin is expressed by cells of the hypothalamic arcuate nucleus in the brainstem. It acts via MOR, influencing appetite as well as sexual behavior. Enkephalin is widely distributed throughout the brain and acts through MOR and DOR. Among them the group includes DTLET (D-Thr2-Leu-enkephalin-Thr, deltakephalin) and DAMGO ([D-Ala ${ }^{2}, \mathrm{~N}-\mathrm{MePhe}^{4}$, Gly-ol]-enkephalin). They act mainly through DORs. Dynorphin acts through KOR and is located in the spinal cord and various parts of the brain including the hypothalamus [2].

The effect of the opioid system on homeostatic and hedonistic control of food consumption is well established and described in detail in Figure 2.

Recent reports also suggest an important role of the endogenous MOR and KOR in the control of energy expenditure and nutrient partitioning.

Numerous studies have established that both systemic and intracerebroventricular administration of general opioid receptor antagonists reduces food intake and body weight in rodent models. In addition, the MOR gene, particularly genotypes of rs1799971 in exon 1 and rs514980 and rs7773995 in intron 1, was positively associated with body mass index (BMI) and obesity [3].

Although the precise molecular mechanism by which opioids decrease food intake is not clearly understood, the central opioid and melanocortin systems interact.

A family of proteins known as melanocortins and their precursors are known to decrease food intake through various mechanisms. Beta-endorphin, one of the precursors, influences mood and food intake; alpha-MSH also reduces food intake. Both substances are encoded by POMC. Interestingly, MORs are expressed in the postsynaptic POMC neurons. These receptors are responsible for the hyperpolarization of these neurons inhibiting their firing. The $\gamma$-aminobutyric acid (GABA) terminals express all three opioid receptor subtypes mentioned above. Their activation inhibits the POMC neurons presynaptically. These pre- and post-synaptic effects of opioids, both the synthetic agonists and the endogenous ones synthesized and secreted by the POMC neurons, exemplify this important interaction between the two systems. Melanocortins act principally through two receptors to reduce food intake: melanocortin receptor 3 and 4 (MC3R and MC4R). Treatment with naloxone helps reduce the stimulation of food intake which is usually enhanced by agouti-related peptide (AgRP), an endogenous antagonist of the melanocortin receptors [4, 5]. Kappa and $\mathrm{mu}$ receptors seem to mediate this interaction because if both are blocked, AgRP-induced food intake is suppressed. However, if only one of these receptors is blocked, there is no change in AgRP action. Agonists of melanocortin receptors have been observed to have an inhibiting action on the orexigenic action of the MOR ligand providing further proof of this interaction [1]. Selective mu-receptor antagonists also blunt the orexigenic action of melanocortin receptor (MC3R/ MC4R) antagonists [6].

It is important to note that the majority of individuals suffering from anorexia nervosa (AN) and bulimia nervosa exhibit antibodies against melanocortin peptide alpha-MSH, which 


\section{Endogenous Opioid Neurotransmitters}

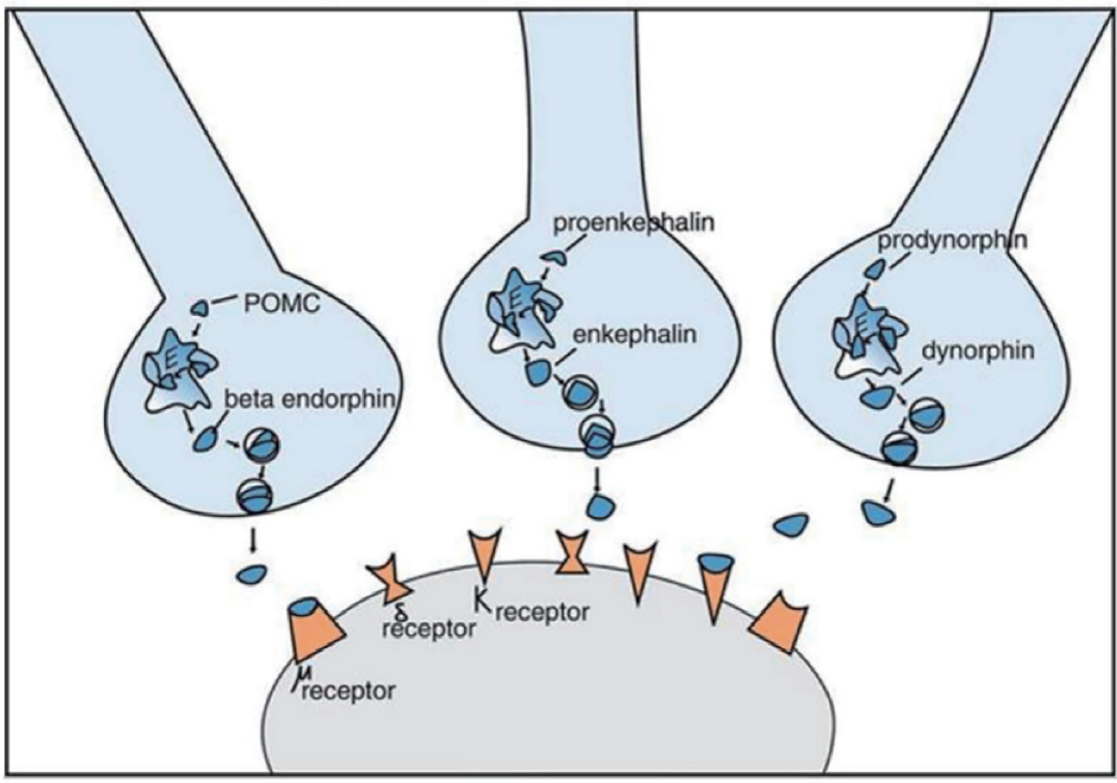

\begin{abstract}
Genes encoding opioidrelated peptides
\end{abstract}

POMC

Prepro-enkephalin
(PENK)
$\begin{aligned} & \text { Prepro-dynorphin } \\ & \text { (PDYN) }\end{aligned}$

\begin{abstract}
Main peptide
beta-endorphin
\end{abstract}

met-enkephalin

met-enkephalin

leu-enkephalin

dynorphin A or B
neoendorphin
Receptor

$\mathrm{Mu}$

Delta

Kappa
Effects

Euphoria

Constipation

Miosis

Analgesia

Respiratory

depression

Euphoria

Analgesia

Dysphoria

Analgesia

Figure 1. Endogenous opioid neurotransmitters. MOR: mu-opioid receptor; DOR: delta-opioid receptor; KOR: kappa-opioid receptor; OPRM1: opioid receptor mu 1; OPRD1: opioid receptor delta 1; OPRK1: opioid receptor kappa 1.

is responsible for decreasing food intake and is influenced by pre- and post-synaptic action of endogenous opioids [7]. In keeping with this, data obtained from experimental models support the hypothesis that opioids are involved in learned and associative appetite processes that influence food selection and acceptance [8].

Neuropeptide Y (NPY) is another important neurotransmitter that acts centrally to mediate energy balance and feeding behavior. Both AgRP and NPY are potent orexigenic factors located in the hypothalamic arcuate nucleus. Some research studies have shown that the opioid system influences the orexigenic effect of NPY. This is because NPY-induced feeding behavior is decreased when naloxone is administered peripherally or centrally [9]. MOR and KOR are mainly involved in facilitating the orexigenic action of NPY. This is supported by the fact that kappa and mu-receptor antagonists are effective in blunting NPY actions while delta-receptor antagonists do not produce this effect [1].

Additional information about food addiction physiology is illustrated in Figure 3.

Orexin A is another orexigenic neuropeptide located in the lateral hypothalamus. Different reports have indicated that orexin-induced feeding behavior is modulated by opioids. Hypothalamic injection of orexin increased enkephalin gene expression in the VTA, paraventricular nucleus and central nucleus of the amygdale, suggesting involvement in its orexigenic effect.

In accordance with this, naltrexone blunted the orexigenic action of orexin A. Interestingly, naltrexone also blocked the effects of orexin A when it was directly administered in the NAcc, indicating that orexin needs to act through areas related to the rewarding properties of food to stimulate feeding behavior [10]. Contrarily, opioids are not mediating the orexigenic effects of melanin-concentrating hormone, another neuropep- 

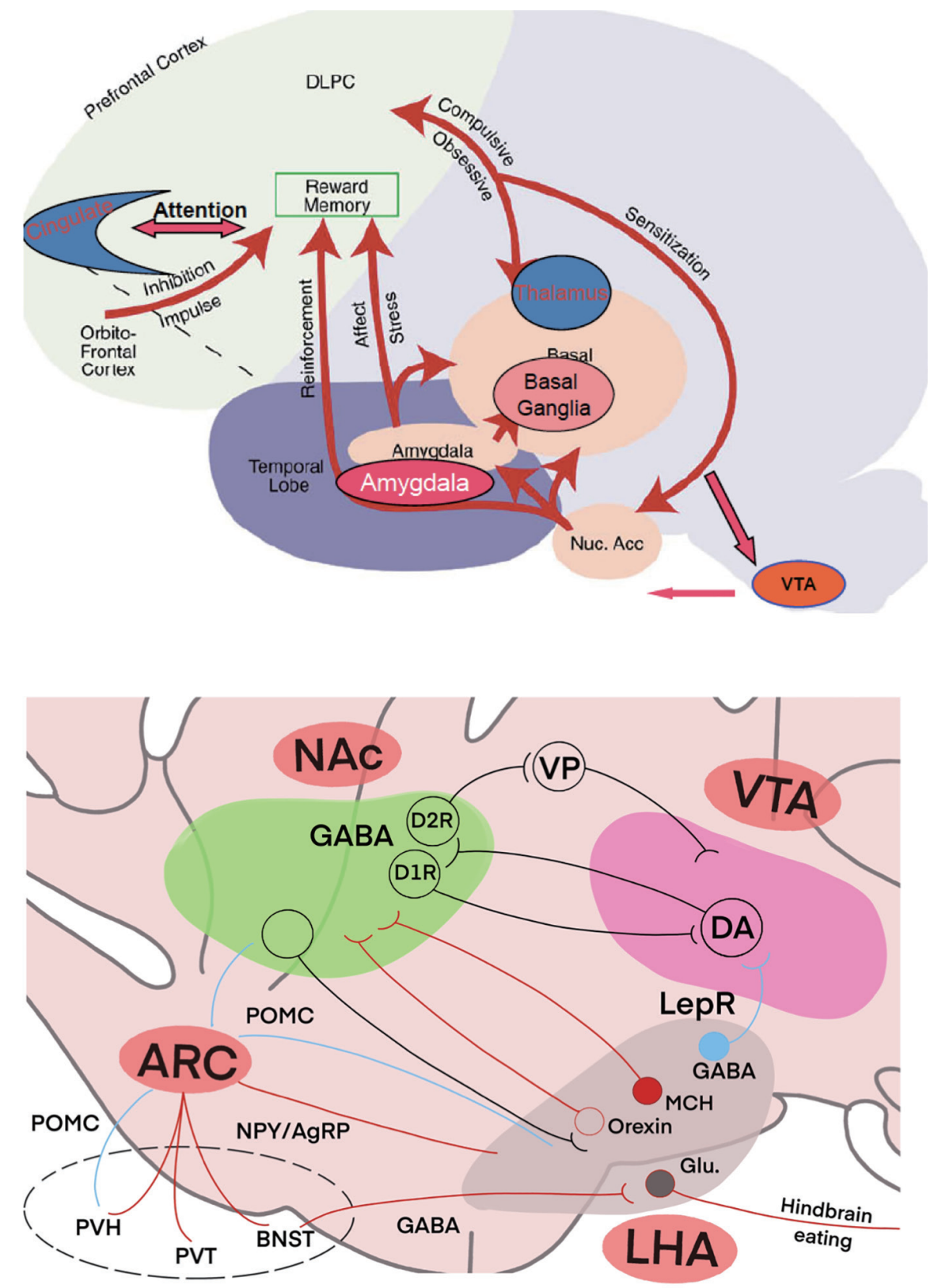

Figure 2. Effects of opioid system on homeostatic and hedonistic control of food intake.

tide located in the lateral hypothalamus.

Another important finding was that the stimulation of high fat intake induced by the administration of DAMGO, a MOR agonist, in the NAcc required intact orexin signaling in the VTA, suggesting that the interaction between the opioid system and orexin modulates both homeostatic and hedonic pathways [11].

In addition to the regulation of food intake by homeostatic signals, opioids play an important role in the hedonic aspects of eating and reward mechanisms, modulating both the palatability of flavored solutions and food. The acquisition of hedonic feeding involves the activation of the mesolimbic dopamine pathway, the dopaminergic projection from the VTA to the NAcc, which is likely the most important mediator of the reward circuitry of food. Endogenous opioids regulate the mesolimbic dopamine pathway at both the levels of the VTA and the NAcc.

Some reports suggest that the effects of opioids on the rewarding properties of food are more potent than their effects on the regulation of homeostatic signals. In this regard, naloxone suppresses intake of a sucrose solution more efficiently than the intake of water and blocks the preference for a saccharin solution $[1,11]$.

Naltrexone administration also produced a similar effect by decreasing preference for sucrose. To the contrary, introduction of an MOR agonist, DAMGO into the NAcc leads to 


\section{Opioid system physiology}
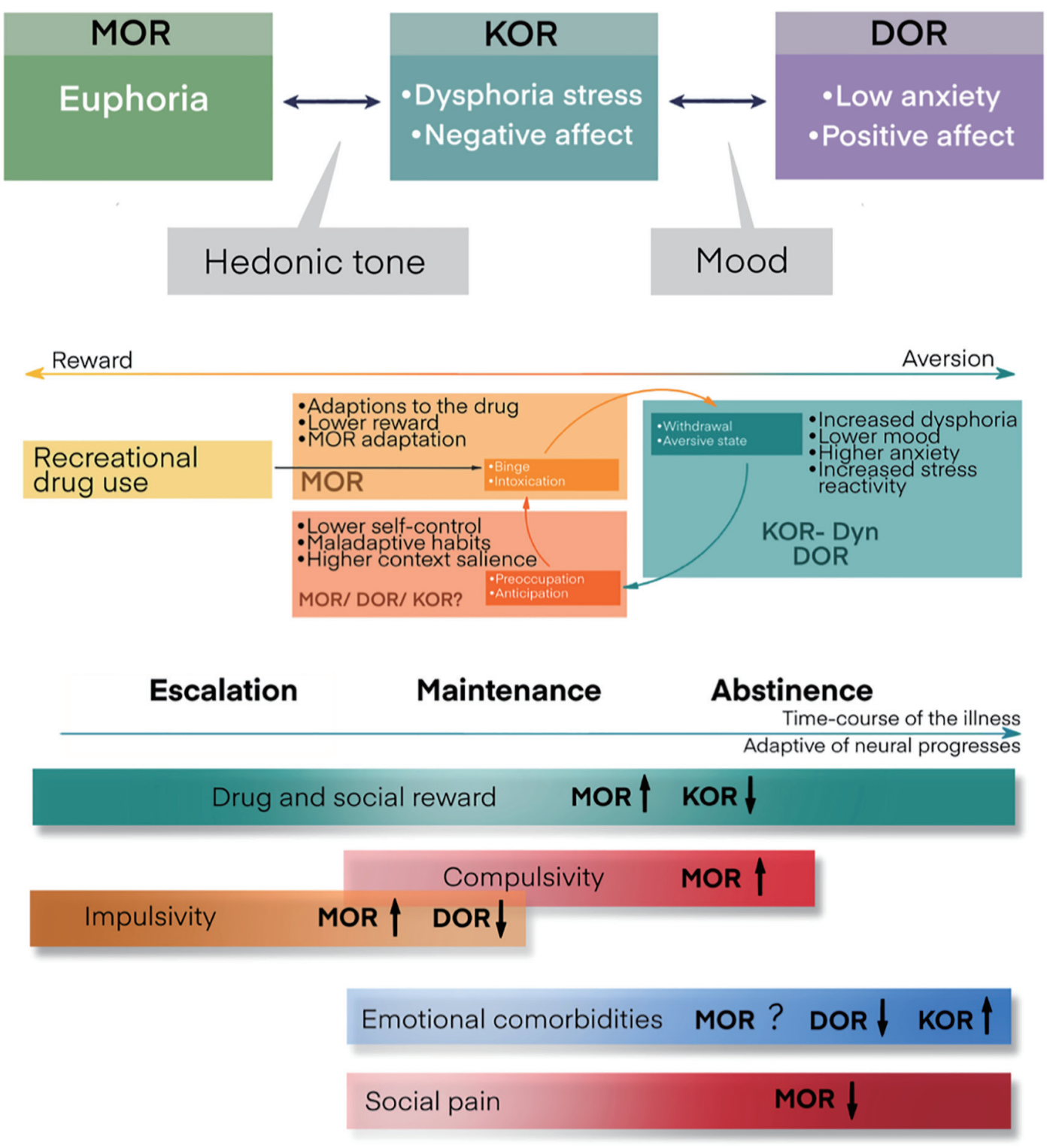

Figure 3. Opiate system and addiction physiology.

an increase in saccharin intake; a similar response is observed when it is injected into the VTA. In human patients with bulimia, there is a reduction in MOR binding in the insular cortex, which has an inverse relationship with fasting. It is still unclear whether this effect reflects a state of craving or if it is due to down-regulation of receptors after fasting [1].

Opioids also modulate the preference for some specific diets, as demonstrated by the fact that the treatment of rodents with naltrexone markedly reduced the intake of a sucrose diet. However, other laboratories have failed to demonstrate the interaction between opioids and food preference [12]. Furthermore, the opioid receptor antagonist naltrexone did not modify ghrelin-induced feeding in the mesolimbic reward pathway. Ghrelin stimulates feeding behavior when injected in the VTA or the NAcc. However, pretreatment with naltrexone in these areas did not blunt the orexigenic action of ghrelin [13].

An important issue that must be addressed is the fact that most of the opioid antagonists have been reported to decrease short-term food intake, but few appear to reduce long-term intake. However, research focused on some synthetic opioid antagonists, the 3,4-dimethyl-4- phenylpiperidines, has demonstrated long-term efficacy. More specifically, LY255582, which in vivo acts as an MOR and KOR antagonist, decreased food intake and body weight over a 7-day period when injected 
Table 1. Pure Opioid Receptor Antagonists

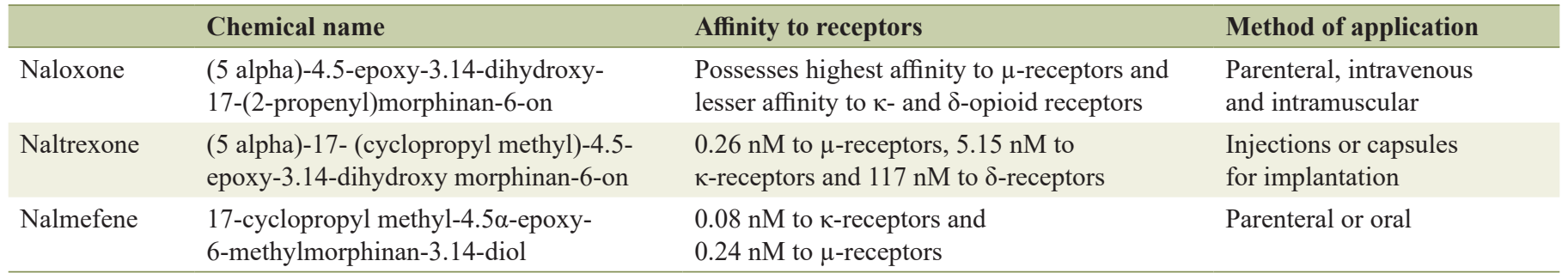

intraventricularly once per day. This compound also decreased food intake and body weight gain when administered subcutaneously to obese rats during a 30-day period of treatment [14].

The substance LY255582 was also found to decrease the intake of lipids and to stimulate lipid utilization resulting in weight loss when given orally for 14 days to rats being fed a high-fat diet. LY255582 also inhibits the intake of a palatable diet after being administered for a period of 4 days by blocking the activation of dopamine neurons located in the NAcc which are usually activated by a highly palatable diet [15].

\section{Opioid Receptor Antagonists in Treating BED and Obesity}

Pharmacological studies of the role of opioids regulating feeding behavior in humans have been limited mainly to general opioid receptor antagonists such as naloxone (intravenously), naltrexone and nalmefene (orally).

In accordance with their interaction with opioid receptors, all opioid preparations are divided into: 1) Pure agonists, including highly active agonists: morphine, trimeperidine (promedol), meperidine, methadone, fentanyl, etc.; poorly active agonists: codeine, propoxyphene, oxycodone, hydrocodone. 2) Mixed agonists-antagonists and partial agonists: buprenorphine (activates predominantly $\mu$-receptors), butorphanol, pentazocine (blocks predominantly $\mu$-receptors and is not used as an analgesic) and tramadol.

Pure opioid receptor antagonists include naloxone, nalmefene, naltrexone, alvimopan and methylnatrexone (Table 1).

These antagonists have also proved to be effective in reducing binge duration in bulimic patients and obese binge eaters [15], although some discordant results have also been reported. Though the reasons for these discrepancies remain unclear, it should be noted that a recent study has documented an increased frequency of the "gain of function" G-allele of the A118G single nucleotide polymorphism of the MOR in obese patients with binge eating. These patients also reported greater scores on a self-report measure of hedonic eating [16].

We will summarize most common studies where opioid antagonists were used in the management of eating and weight disorders. All these studies were carried out in a low number of normal-weight patients, but most of them found a decrease in short-term food intake, while no significant effects were observed on hunger.

The decrease in food intake was very consistent, with a range of $11-29 \%$, suggesting a clear role for opioid receptors in human feeding behavior. About $19 \%$ of subjects reported nausea after the administration of naltrexone, compared to $9 \%$ receiving placebo [17]. The actions of naloxone and naltrexone on feeding behavior have also been studied in obese patients. Both medications were able to inhibit food intake, and some of those obese subjects also reported a decrease in hunger [18].

According to Sinclair et al, treatment of eating disorders requires application of a method of treatment known as selective extinction. In this method, treatment targets weakening of the disorder while enhancing healthy eating habits. However, selective extinction cannot be used in isolation. In this method, sessions of extinction are carried out with the goal of weakening the disorder, while an opiate antagonist is used to block the opioid system. The use of both the pharmacological agent and sessions of learning is done through interspersing such that when the patient is learning how to develop proper eating habits, they are free from the opioid antagonist [19].

Nalmefene not only attenuates binge behavior but also increases food intake of the less preferred diet [1]. These effects are likely mediated by inhibiting MORs in the VTA, leading to disinhibition of GABAergic interneurons and subsequently to decreased dopamine release in the NAcc [20].

The essence of interspersing lessons on extinction and use of opioid antagonists is to allow for a washout period between each dose of the opioid antagonist from the body. During this washout period, the patient should not eat regardless of whether they have an eating disorder or healthy eating practices. The implication of this is that opioid antagonists that have long duration of action or half-life such as nalmefene and naltrexone should not be used since they have long washout periods. Instead, naloxone that has a half-life of approximately $1 \mathrm{~h}$ should be utilized since it will support a shorter yet essential washout period. Additionally, the use of naloxone in treating bulimia is advantageous compared to other opioid receptor blockers since purging, which is common with this eating disorder, does not affect its dosing. Furthermore, its administration neither alters activity of the gastrointestinal system nor interferes with responses the patient gives with respect to their eating habits due to extinction learning [19].

Moore et al conducted a study to determine the effect that treating patients with $\mathrm{AN}$ with naloxone had with respect to weight gain and lipolysis. Patients' blood was tested for levels of both beta-hydroxybutyrate and non-esterified fatty acid (NEFA), which are vital indicators of ketogenesis and lipolysis, respectively [21]. They found that naloxone contributed to a reduction in the levels of both beta-hydroxybutyrate and NEFA. Additionally, the subjects showed significant gain in 
body weight during the period of infusion with naloxone compared to the period prior to and after the infusion. In their conclusion, they indicated that naloxone has antilipolytic effect that was responsible for weight gain as all the formed lipids were stored instead of being broken down [21].

According to Stolar, opiates have the effect of either increasing or decreasing the eating habits of individuals. Naloxone lowers hyperphagia that is induced by diazepam in animals that have been satiated. With the induced satiety, the animal terminates their feeding. Additionally, naloxone lowers eating stimulants that come through olfactory, gustatory, thermal and visual senses, and blocks rewards that animals get from food, including its palatability. When rats were stressed through mild pinching, their food consumption increased. According to Stolar, naloxone can be used to prevent eating induced by such stress in these rats [20].

According to Blasio et al, naltrexone, when administered systemically and into the NAcc, reduced a fixed ratio 1 (FR1) response for food and motivation to eat under a progressive ratio in both groups of rats; conversely, when administered into themedial prefrontal cortex (mPFC), the effects were highly selective for binge eating rats. Moreover, they found a twofold increase in POMC and a 50\% reduction in pro-dynorphin (PDyn) gene expression in the $\mathrm{mPFC}$ of rats on a sugary diet, when compared to control rats; however, no changes were observed in the NAcc. They suggested that neuroadaptations of the opioid system in the mPFC occur following intermittent access to highly palatable or sugary food, which may be responsible for the development of binge-like eating behavior [22].

In another study by Drewnowski et al, researchers set out to test the hypothesis that naloxone could be used to reduce the consumption of foods that were high in fat and sugar by females who either obese or lean and had BED. They administered, through intravenous infusion, butorphanol that is an agonist at opioid receptors, naloxone and normal saline as placebo to 16 females who were obese based on their BMI and 25 females who had normal weight. During the period of administering these drugs, participants were exposed to 20 different dairy products that had been sweetened and eight different snacks that contained differing levels of both fat and sugar. In their findings, the researchers demonstrated that naloxone caused suppression of participants' desire to consume foods that had high fat and sugar content. However, this was only observed with participants who were binge eaters. Additionally, it was noted that obese participants and non-binge eaters showed no significant difference in eating habits when they were given naloxone. Butorphanol, on the other hand, showed no statistically or clinically significant difference in hedonic response that participants demonstrated on their eating habits in any of the two subject categories [23].

In another study by Holtzman, the researcher set out to demonstrate that naloxone suppressed appetitive habit in rats. The researcher administered naloxone and morphine to rats that had been grouped based on the three objectives. Findings of this study indicated that naloxone suppressed both drinking of water and eating in the subjects in a dose-dependent manner. They also indicated that suppression of intake of water was not affected by physical dependence on morphine when these rats were given naloxone. In his conclusion, the researcher indicated that naloxone significantly suppressed both feeding and drinking habits of rats. However, the conclusion indicated that this suppression could not be fully explained by naloxone interacting with receptors within the opioid system of the body [24].

Naltrexone is classified as an antagonist of opioids that readily binds with great affinity to $\mu$-receptors. Mice that have been engineered in a way that they do not possess the $\mu$-receptors have been shown to resist obesity despite being subjected to a diet that has high content of fat. When administered chronically, naltrexone is known to increase the amount of POMC messenger ribonucleic acid (RNA). This increase causes restoration of POMC neuronal activity and satiety due to melanocortin. By blocking the actions of beta-endorphins located at the $\mu$-receptors, naltrexone prevents autoinhibition of neurons of POMC [25].

Acute administration of naltrexone has the effect of influencing both eating habits and the reward system activity. It inhibits the rising levels of dopamine within the NAcc that occurs when food is ingested thus reducing the amount of food that an individual consumes. It also influences food seeking habits of an individual and binge eating behavior. When injected directly into the VTA and NAcc that combined make up the reward system of the body, this drug reduces preferences individuals have for foods considered to be greatly palatable. Additionally, this drug influences the manner in which one expresses their preference for flavors and diets that are palatable after going through a period of abstention. It also influences the likelihood of an individual to binge eat. Therefore, naltrexone generates a reduction in intake of food by animals especially in situations where they have been chronically exposed to intake of high-fat food [25].

Giuliano et al studied the effects of GSK1521498, a novel $\mu$ opioid receptor antagonist, on food seeking behavior and on binge-like eating of a highly preferred chocolate diet. Both groups of rats were treated with GSK1521498 or naltrexone. Both compounds reduced binge-like palatable food hyperphagia. However, GSK1521498 reduced the impact of high hedonic value on ingestion more specifically than NTX, abolishing anticipatory chow hypophagia. GSK1521498 also dose-dependently reduced food seeking both before and after food ingestion, whereas NTX reduced food seeking only after food ingestion. Thus, while both drugs affected the hedonic value of the preferred food, GSK1521498 also directly decreased incentive motivation for chocolate [26].

In another review, the authors indicate that naltrexone, when used as monotherapy, is associated with minimal loss in body weight. However, they mention that this drug has the potential for use in treating patients with obesity when combined with bupropion, which acts as an activator of POMC. Naltrexone showed a reduction in body weight by an average of $4.7 \%$. These authors indicate that the two medications that are part of the recommended regimen for losing and maintaining weight generate a reduction in body weight of between $5 \%$ and $10 \%$ after 1 year of treatment [27].

Bertino et al carried out a double-blind study with the aim of testing the hypothesis that a reduction in the intake of food that occurs when opioid blockers are administered is due to 
the ability of these antagonists to lower taste features that are pleasant. The subjects for this study were 18 college students, all of which were males. They were asked to rate both pleasantness and intensity of soup that had different concentrations of sodium chloride and sucrose contained in Kool-Aid. This was done after they had been given $50 \mathrm{mg}$ of naltrexone and a control group given a placebo. The researchers found out that pleasantness the subjects felt when they tasted soup that was salted increased following administration of a placebo especially when lunchtime was approaching. However, the category of subjects that had been put on naltrexone reported an unchanged level of pleasantness in this soup. Additionally, they indicated that naltrexone inhibited an increase in levels of hunger. They also found the category of subjects that had been given naltrexone consumed less calories compared to the placebo group, to the extent of $-500 \mathrm{kcal}$. They concluded that a reduction in hunger was responsible for reduction in intake of food that was induced by naltrexone [17].

In another randomized controlled trial (RCT) that was double-blind by Malcolm et al, the researchers set out to examine the efficacy that naltrexone had in promoting loss of weight. Researchers found that subjects who were on naltrexone had a loss of body weight that was averagely $1.8 \mathrm{~kg}$ while the group that was on placebo lost an average of $1.5 \mathrm{~kg}$ of body weight. The researchers concluded that the difference in response to naltrexone and placebo was not clinically significant [28].

The anti-obesity medications reviewed by researchers at Weill Cornell Medical College included Food and Drug Administration (FDA)-approved medicines for chronic weight management, FDA-approved medicines for short-term use in weight management and off-label use of medicines for weight control. They explored the use of a combination of naltrexone and bupropion in the treatment of obesity. They indicated that naltrexone acted by diminishing feedback that is autoinhibitory on neurons that are activated through the action of bupropion. As such, this combination generates weight loss that is sustained. This combination exists in strengths of $90 \mathrm{mg}$ of bupropion and $8 \mathrm{mg}$ of naltrexone. They explored placebocontrolled double-blind trials that were conducted to assess the efficacy of this combination and concluded that they were effective when combined with lifestyle modification practices and dietary changes [29].

Selleck and Baldo (2017) performed a systematic review of literature with the objective of describing findings of recent studies regarding actions on motivation that opioids had within the prefrontal cortex and its effect on feeding. They found that eating was induced by the action of opioids in the prefrontal cortex and NAcc through enhancement of reactivity to taste. They concluded that opioids acted in a direct manner through the NAcc in facilitating motivation by an individual to eat and hedonics of taste [30].

Yeomans and Gray (1996) carried out a randomized placebo-controlled double-blind study to determine the effects that naltrexone has on intake of food and its pleasantness. They used 16 subjects and found that naltrexone produced significant reduction in pleasantness of food and its intake when a comparison was made with either the baseline or the placebo group. However, they noted that the effect of naltrexone was not uniform across different types of foods. Its effects were pronounced with foods that had high content of proteins, fats and sucrose content. Additionally, the amount of food intake was also reduced but sweetness and saltiness of food was not affected by naltrexone [31].

In September 2014, the US FDA approved Contrave, a combination of bupropion and naltrexone, for obesity management. It is also intended for patients who are not suffering from obesity but have excessive body weight and are at high risk of cardiovascular events.

The given preparation appeared after it was found that naltrexone blocks $\beta$-endorphin-mediated inhibition of POMC, i.e. a prohormone that supports a-melanocyte-stimulating hormone (a-MSH) secretion. Naltrexone is the antagonist of opioid receptors sold in the US for treating drug and alcohol dependence. Bupropion (via dopamine receptors) meanwhile activates POMC neurons and enforces the a-MSH anorexigenic neuropeptide secretion in the hypothalamus and is an inhibitor of the dopamine and norepinephrine reuptake being administered as an antidepressant and a means to stop smoking $[32,33]$.

Bupropion inhibits dopamine and norepinephrine reuptake, thus increasing the level of these neurotransmitters in the synapse. Interestingly, it is chemically linked to amphetamines. All amphetamines and their related products (e.g. phentermine and diethylpropion amphetamine congeners) reduce appetite. The mechanism is not clear; however, it could be connected with their action in central noradrenergic and dopaminergic systems that are able to tightly compress the food reward. It is possible that they impose a direct effect on the regulation of appetite in the hypothalamus, but this action is not sufficiently described. We believe that the bupropion-naltrexone combination acts on the food intake motivation processes (dopamine effect) and on the pleasure/taste qualities of food (opioid effect) in order to initiate weight loss and maintain it for a longer period of time, disabling the natural compensatory mechanisms involved in the typical plateau stage of loss of weight [34]. During clinical trials, patients taking Contrave in combination with diet and physical exercises, lost more weight than patients taking placebo and adhering to the same diet and physical exercises.

In 2013, a clinical study was published devoted to evaluation of bupropion and naltrexone effect on depressive symptoms and body weight alterations with patients having excessive weight or obesity and major depressive disorder. Female patients $(n=25)$ were included into the 24-week open label study and were treated with naltrexone sustained release (SR) $32 \mathrm{mg} /$ day and bupropion SR $360 \mathrm{mg} /$ day. Significant improvement in the Montgomery-Asberg depression rating scale indicators was observed; loss of weight by $-4.0 \%$ at week 12 and by $-5.3 \%$ at week 24 was demonstrated using the intent-totreat (ITT) analysis [35].

Pennington Biomedical's research team conducted an extensive systematic literature review to assess eating habits that were reward-induced. They indicated that control that is homeostatic of eating habits had a linkage to reward systems in a manner that was inextricable. The implication of this is that appetite is an outcome of a response that is coordinated by the balance of energy [36]. They indicated that when food is 
abundant in an environment, and as such choices are numerous, individuals are motivated to eat from a wide pool, and this plays an important role in them overeating. Additionally, they indicated that responses individuals had to food intake were related to various cues. It is such a response and homeostatic control that the combination of naltrexone and bupropion targets. Similar findings were described by Mason et al, who demonstrated that naltrexone produced blockade of the opiate system and thus lowered eating that was driven by anticipated reward and craving for food [37].

Studying the currently published reports, it could be concluded that the effectiveness of bupropion plus naltrexone in the treatment of obesity is clinically moderate, but statistically significant. However, in the majority of studies loss of weight of at least 5\% was achieved without behavioral changes among patients. This weight reduction is similar to that achieved using other preparations approved in the treatment of obesity, but not well maintained. Table 2 summarizes all reports included in this review and provides additional information $[17,19-21$, 23-27, 28-32, 36, 37].

\section{Psychological Interventions as Augmentation Therapy in BEDs}

Inadequate treatment of earlier episodes of binge eating may lead to kindling and sensitization at the receptor and synaptic levels leading to the development of resistance [18]. Standard approaches to pharmacology include the optimization of the current treatment regimen and within-class or between-class switching. Underdosing and inadequate length of treatment with naltrexone-bupropion cause resistance to treatment. Other factors include poor compliance, individual differences in drug metabolism, nutritional status and inadequate social support.

As we discussed earlier, opioid antagonists have been shown to decrease short-term food intake and decrease the pleasurable nature of palatable foods and have been long considered in weight loss therapy [18]. However, the results of clinical trials have been mixed, with a few suggesting that opioid antagonists failed to yield any significant effect in the treatment of obese binge eaters in long-term perspective [38]. Augmentation, unlike adjunct treatments for BED and obesity, has not been studied, but may enhance outcomes when included within specialist treatment programs. Augmentation using psychological interventions with established efficacy may also be referred to as combination therapy. We therefore considered these approaches hypothesizing that augmentation with psychotherapy might play an important role in treating binge eaters because a chronic condition like BED, especially combined with affective disorder, is less likely to respond to medication than is an acute condition. This suggests a greater need for adjunctive treatment strategies.

BED and obesity have many similar characteristics, but are not necessarily related. Most obese people do not have BED; however, most people with BED are either overweight or obese. Combined behavior therapy and pharmacotherapy may be considered the optimal strategy for many obese patients. Surprisingly, combining medications with psychotherapy in- terventions fails to significantly decrease binge outcomes, although specific medications may enhance weight loss achieved with CBT and behavioral weight loss. Contrave seems to be an interesting preparation in reducing appetite; however, treatment with medications alone is usually not enough; it requires behavioral changes of the patient in order to get full recovery from the disorder.

Providing additional augmentation treatment following psychopharmacotherapy could help reduce these residual symptoms. For example, to compensate for naltrexone's lack of pharmacological reward, psychological intervention could be used to reward and thus enhance naltrexone compliance. Similarly, individual and group therapy could be used to provide incentives for retention and encouragement to persist with treatment despite recurrent binging episodes. Other forms of psychosocial support include family therapy through attachment approach and 12-step self-help programs addressing the problem as an addiction.

Several techniques for coping with binge eating were studied in several RCTs, but none of them were evaluated as an augmentation strategy in treatment with opiate antagonists. The most commonly recommended techniques are included in Table 3.

Generally, psychotherapy modalities make attempts to enhance patients' self-representation, ability to solve problems and their capacity to regulate affective states. Anatomically, the areas in the human brain that carry out these functions include the ventrolateral prefrontal cortex, amygdala, insular cortex, precuneus, posterior cingulate cortex, dorsal and ventral subregions of the medial prefrontal cortex, dorsal anterior cingulate cortex, ventral anterior cingulate cortex and the dorsolateral prefrontal cortex. Despite knowledge of brain areas involved, the understanding of brain-based processes altered by psychotherapy is still low.

CBT for eating disorders is a gold standard of treatment [39]. CBT could be used as an augmentation strategy. The overall goal of CBT is to alter thinking and behavior patterns that are associated with undesired behavior. The therapy approach works by an overall focus on changing individuals' beliefs, images and attitudes that relate to certain behaviors. This approach showed better improvements over family therapy at 6 months, but not 12 . For all studies examining CBT, the protocol for BEDs includes three stages: 1) Establish rapport, increase motivation for treatment; 2) Address distorted cognitions surrounding food, eating, weight and shape; 3) Consolidate treatment gains and develop a relapse prevention plan.

Opioid addiction treatment studies have found that naltrexone along with psychosocial or behavioral therapies, such as CBT have led to positive outcomes [40]. Binge eaters would be expected to demonstrate comparable outcomes. In depressed patients, benefit of CBT as augmentation is slower (by up to 3 weeks) compared to augmentation with medication. This approach has never been studied as augmentation in eating disorder patients on combined psychotropic therapy. Nevertheless, research indicates that CBT is potent treatment for the majority of outpatients with an eating disorder [39] and may be considered as augmentation strategy.

One major advantage of CBT is that it produces results in a relatively shorter time than other treatment approaches. Ide- 


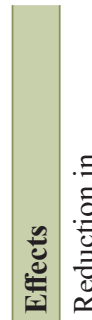

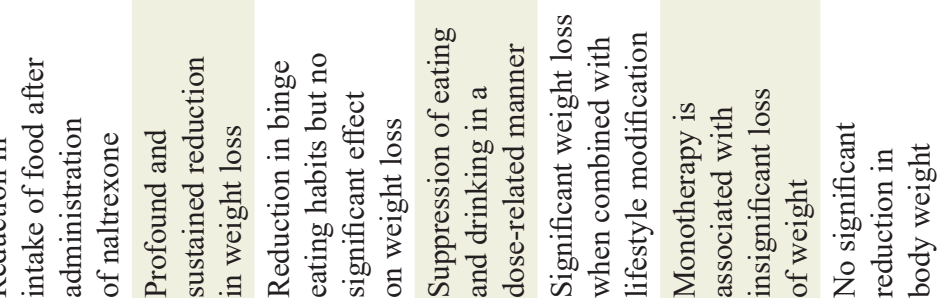
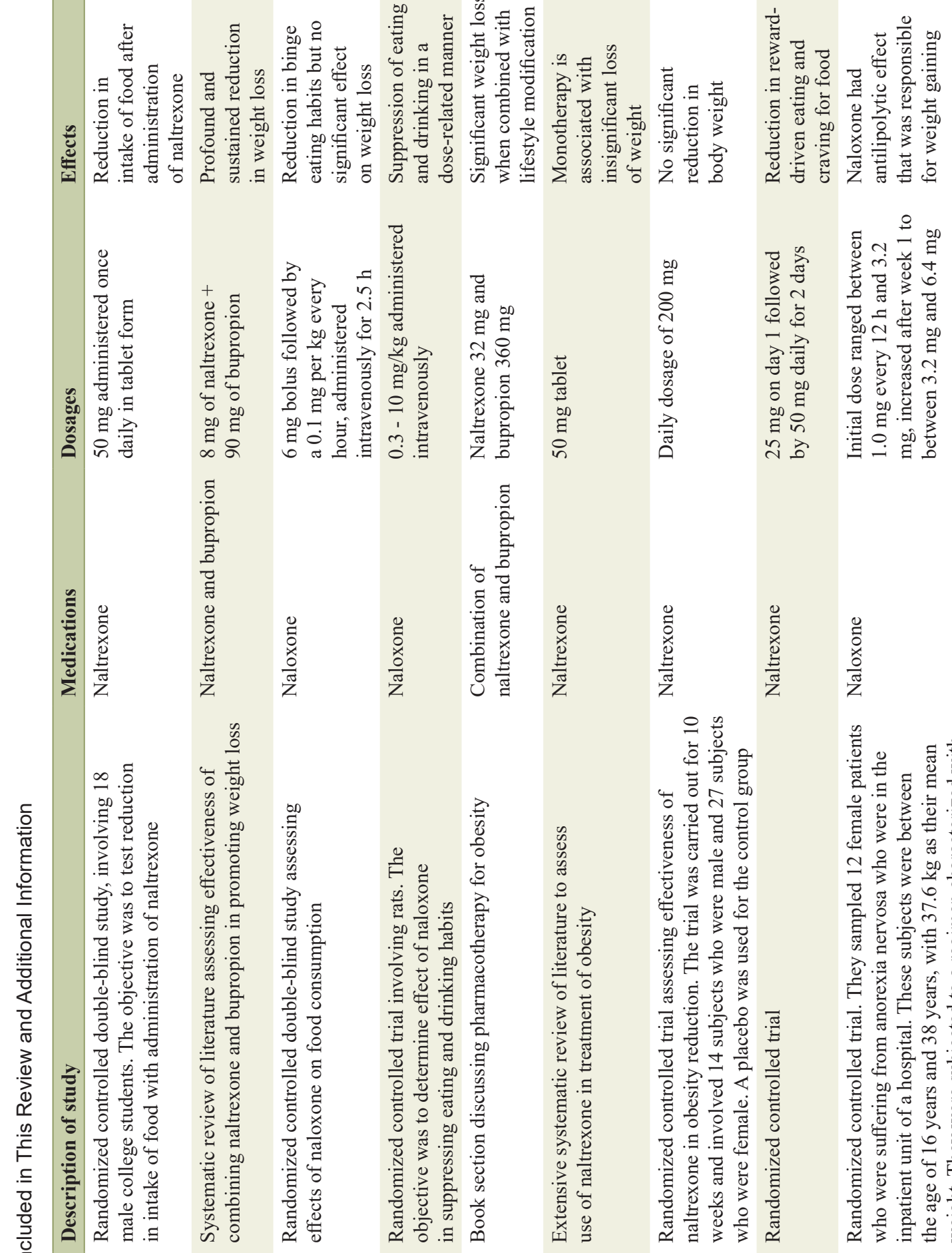

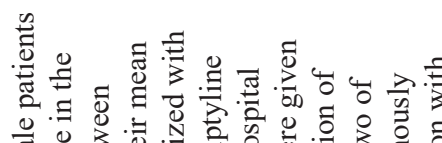

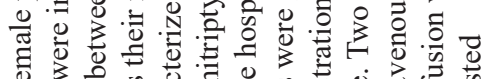
巴

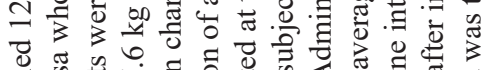

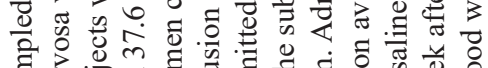

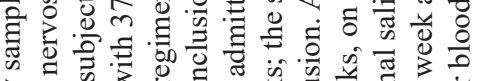
希.

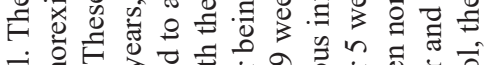

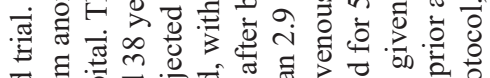

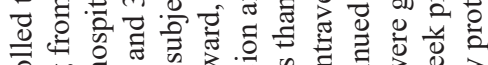
要

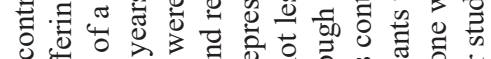

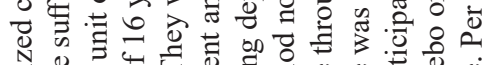
光

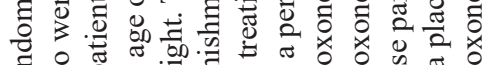

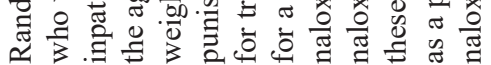

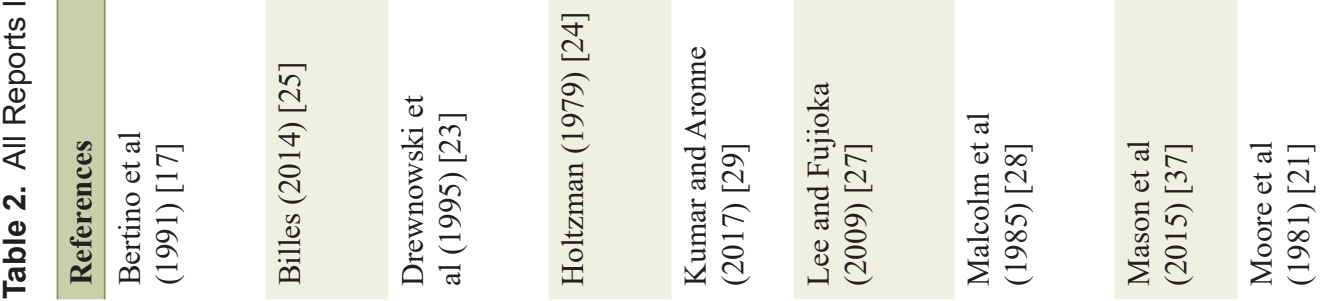



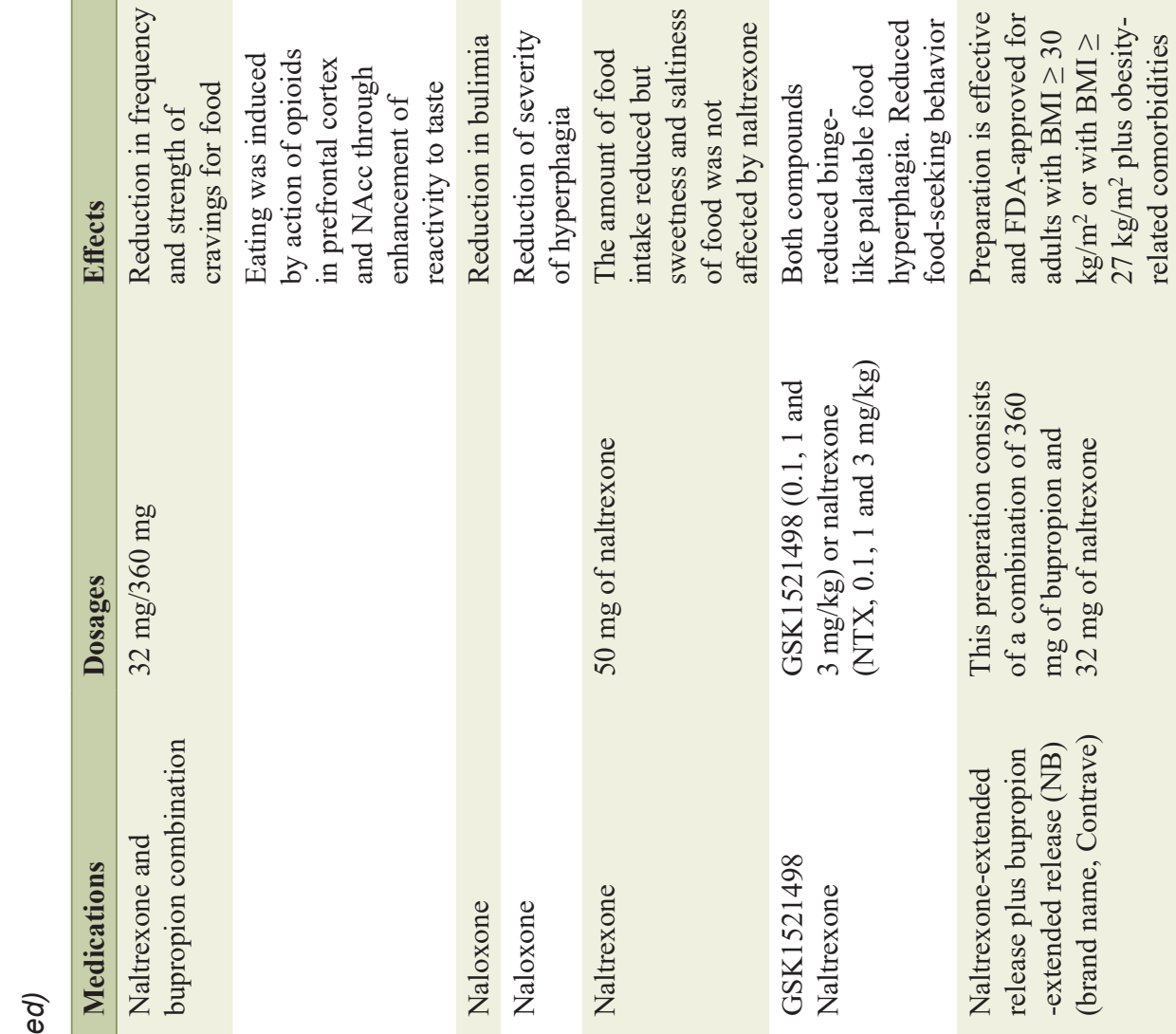

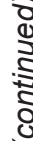

든

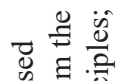

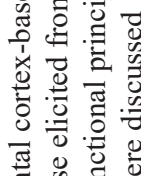

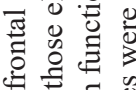

흔

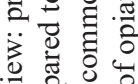

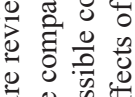

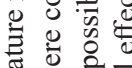

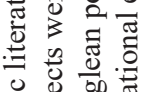

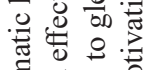

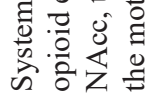

응

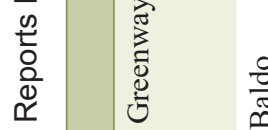

¿

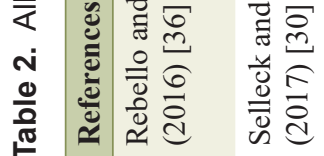

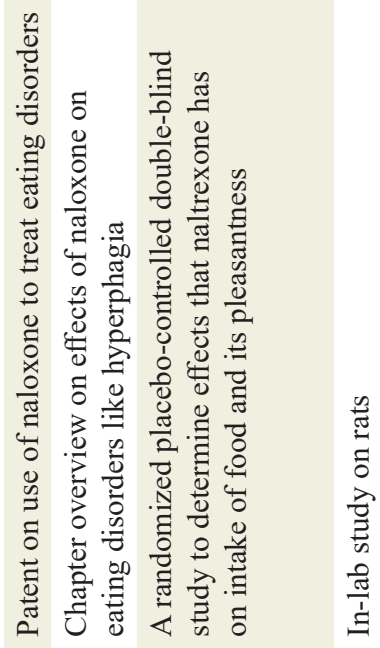

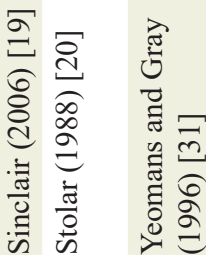

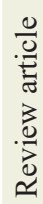

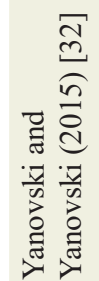

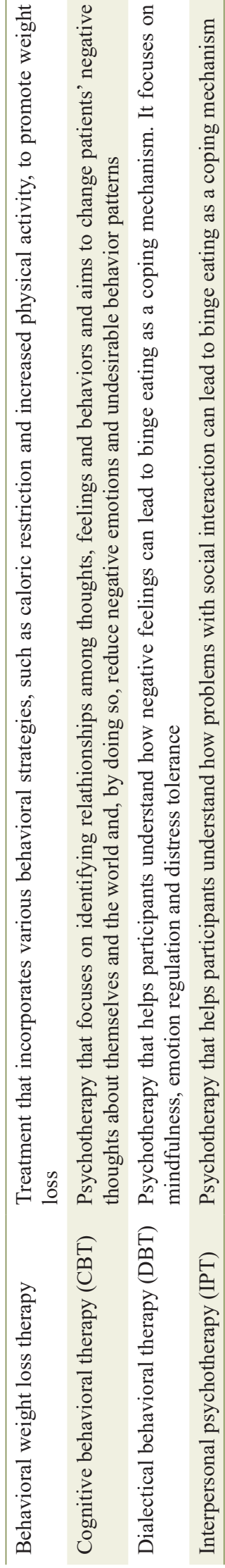


ally, the therapy works within $5-10$ sessions. Moreover, CBT is very instructive, well structured, adaptive and cross-cultural. By correcting problematic underlying assumptions, CBT creates long-term results since the cause of the problem (maladaptive thinking pattern) is corrected.

The challenge with CBT, as with medication, is compliance. Challenging the belief system of an individual who strongly believes in their thoughts can lead to ambivalence. This ambivalence may lead to reduced motivation to continue treatment. At the same time, CBT is not often thought of as a quick fix, and only serves as a guideline towards recovery [41]. With this, there are multiple cases of relapse. People with underlying learning difficulties may also find it hard to concentrate and learn from therapy sessions. This way, they may often gain little from CBT. Furthermore, critics of this therapeutic approach also argue that the focus of this method is often on the current problem and may thus overlook underlying conditions such as mental health conditions that may be secondary to childhood traumas and related stress. Additionally, this approach only focuses on individual abilities to alter and moderate their own behaviors and ignores the external modifiers of behavior such as family and points of exposure that contribute to the overall development and progression of the condition [42].

There is a possibility of an increased likelihood of achieving a patient's goals with CBT by enhancing repeated preconscious (masked) priming of personalized positive cognitions. The priming procedure was associated with decreased negative cognitions and increased positive cognitions on the screening questionnaires, such effects were not observed when the control procedure was applied. Applying the priming procedure immediately after group therapy leads to a higher likelihood of achieving cognitive changes in patients with eating disorders. This could be seen as an augmentation strategy for therapy.

The willingness of the patient to get better is key, and CBT has proven to be successful in treatment of BED. However, authors concluded that CBT does not solve all problems in the eating disorders. This was because only slightly more than half of the individuals starting the treatment achieved a full and long-lasting recovery [43]. Another study on CBT entailed a comparison of two transdiagnostic CBT treatments among outpatients who were diagnosed with eating disorders. According to this study, the two transdiagnostic options in the treatments were suitable in managing the majority of the patients. However, the simpler version was found to be the better adopted method among the patients [44].

There is also the use of enhanced cognitive behavioral therapy (CBT-E). This method is based on the transdiagnostic theory that was derived from CBT. This form of CBT is designed as such to be delivered to an individual with BED on an individual basis [45].

A study carried out by the Research Triangle Institute of the University of North Carolina Evidence-based Center reviewed the efficacy among treatment options of BED. This study looked at the harms associated with the treatments, factors of the treatment efficacy and the differences in the treatment outcomes associated with the different sociodemographic characteristics. Six major databases on the treatment of BED were searched. This was done following an inclusion-exclusion criterion focused on the eating habits and psychological outcomes. Twenty-six studies were done which included medication only treatment, behavioral intervention plus medication and behavioral treatments alone. Evidence for behavioral and medication interventions used was moderate. Evidence for other interventions and self-help among these patients was weak. There was strong evidence on the harm associated with the treatments. There were no differences in the outcomes based on sociodemographic characteristics of the patients included. The study proved that individual or group CBT reduces BED. Use of CBT among these patients also showed improvement in abstinence rate up to around 4 months after initiation of treatment. However, use of CBT did not lead to reduction of weight among these patients [46].

Additionally, in the treatment of eating disorders among the youth, a study by Wilfley suggested that their outcomes depend on parental involvement. According to this RCT among children and adolescents, CBT was used together with negative behavior cycle breaking. The article reviews current supported treatments among the youth. The rationale included the extension of therapy to include the family, the immediate community and peer group of the individual. Using this, there was promotion of behavior change, minimizing relapse of the disease, therefore promoting a long healthy life [47].

Interpersonal psychotherapy (IPT), a structured form of psychodynamic therapy, has also been used effectively in treating eating disorders [39]. It is a time-limited treatment directed at improving interpersonal skills that may have contributed to the development of eating disorders. IPT focuses on resolving interpersonal problems positing that more effective interpersonal functioning will lead to healthier eating habits and attitudes. The primary period of IPT distinguishes explicit relational issues that currently affect the patient and fine-tuning which of the identified areas needs the most focus. In the second period of IPT for eating disorders, the therapist urges the patient to lead the facilitation of change in the relational domain. The third stage covers upkeep of relational gains and backslide avoidance.

Among the greatest of limitations that come with the application of IPT in the management of both eating and substance abuse disorders is that it is often difficult for clients who have been through other forms of psychotherapy to fit into the process. With the change to IPT, there is often a need to achieve results within a fixed time frame, and this may often pose greater problems with the clients being not receptive to the stringent measures of the therapy process.

A study on IPT performed in 1993 showed that IPT takes a longer time in the management of BED and bulimia nervosa compared to CBT. In the study, it took about $8-12$ months longer with IPT to get similar clinical outcome achieved when the patients were managed using CBT [48]. The conclusion made was that treatment of the eating disorders would be achieved without focus on the patient's personal attributes. Patients managed using CBT had a better clinical outcome compared to those managed using IPT. Overall, IPT may be considered as an alternative to CBT, but the response is less pronounced and slower to be expressed $[39,48]$. Figure 4 provides additional details on this matter.

Dialectical behavior therapy (DBT) is a form of subjective behavioral therapy similar to CBT. Its fundamental objec- 
Response to Cognitive Behavior Therapy (CBT)

or Interpersonal Psychotherapy (IPT)

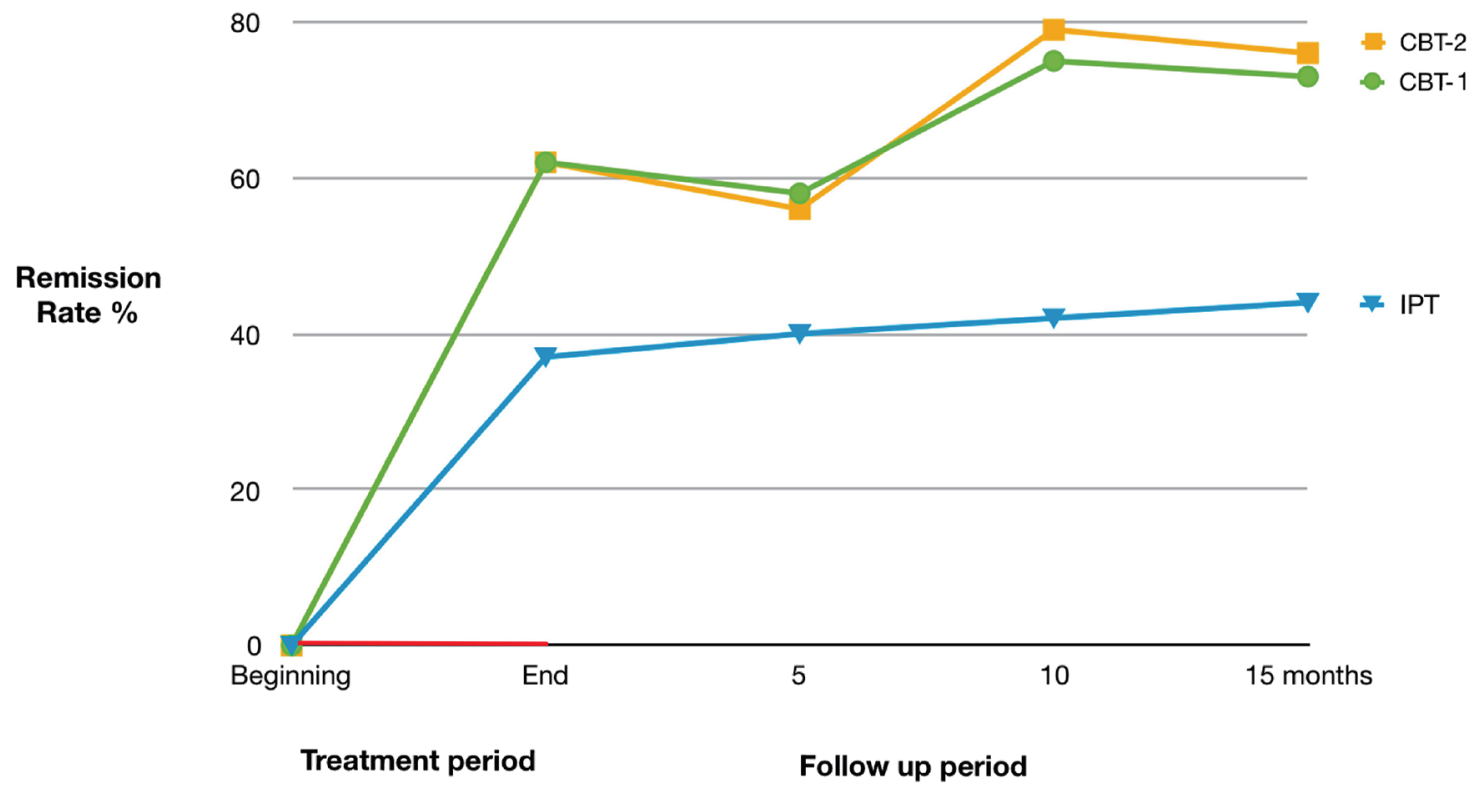

Figure 4. Response to cognitive behavioral therapy (CBT-E) or interpersonal psychotherapy (IPT).

tives are to show individuals how to live in the moment, adapt soundly to pressure, direct feelings and improve associations with others. It was initially planned for individuals with BPD yet has since been adjusted for different conditions where the patients display self-destructive behaviors, for example, dietary problems and substance misuse. In binge eating, DBT has been cited as a form of integrated behavioral therapy that combines CBT and other supportive therapies. It is regarded as extremely efficient and efficacious in the management of various eating disorders, with a special emphasis on BED. Rather than seeking to only change behavior, the approach seeks to help patients accept their conditions and cope better, while in the process, moving away from the undesired behavior. This therapy urges and trains patients to be present in the moment and accept the associated emotions. With the recognition of the trigger emotions, the patient is better able to deal with the unconscious process that prompts them towards binge eating episodes [49]. Table 4 outlines five major functions of DBT in management of binge eating episodes. Similar approaches may be used as an augmentation strategy.

While there are little research data on the effectiveness of DBT in the management of various conditions, existing research findings indicate that DBT has almost equal outcomes for both substance abuse and eating disorders [50]. Particularly, a 2001 comparative study demonstrated that up to $89 \%$ of the overall participants who underwent dialectical therapy for different conditions showed positive correlations in all of their conditions, with this percentage being free of their symptoms by the end of the study period [50].

Behavioral family systems therapy (BFST) has been used effectively in helping patients with AN. A similar approach could be used in controlling binge eating episodes in BED patients. Unlike a family-oriented approach, BFST does not focus on empowering parents to use their own intuition to facilitate changes in meals and food choice - parents must work with a dietitian in order to understand the concept of this therapy.

Parents should be in control of overeating, but sessions supposed to identify cognitions that are underlying eating disorders. Moreover, BFST may improve family relationships and treatment adherence significantly, while reducing $\mathrm{HbAlc}$, particularly among adolescents with poor metabolic control [51]. Clinical efficacy of BFST in obese and diabetic patients may be useful in further clinical studies on BED.

\section{Other Contemporary Treatment Modalities}

There is an increasing number of psychosocial and promotional approaches that address individual, family and social risk factors, which are promising for the development of positive self-image and self-efficacy in patients with BED.

There is evidence to support the fact that mindful eating helps in the treatment of BED as well as obesity. Practicing mindfulness techniques has been shown to lead to reductions in shape and weight concerns, thin-ideal internalization, dietary restraint, psychosocial impairment and eating disorder symptoms. Several techniques can be used to help individuals with eating disorders who have been overwhelmed by their thoughts and are likely to engage in self-destructive behaviors.

Mindfulness-based therapies have received initial support 
Table 4. Dialectical Behavior Therapy for Binge Eating Disorder

\begin{tabular}{ll}
\hline Function & Example \\
\hline $\begin{array}{l}\text { 1. Enhance capabilities } \\
\text { 2. Increase motivation }\end{array}$ & $\begin{array}{l}\text { Behavioral skills training including modeling, behavioral rehearsal, psychoeducation regarding binging, } \\
\text { feedback, homework assignments }\end{array}$ \\
$\begin{array}{l}\text { Individual sessions: behavioral assessment including triggers and consequences of binge eating } \\
\text { behavior, contingency management. Exposure-based strategies and cognitive modification approach are } \\
\text { also applicable }\end{array}$ \\
$\begin{array}{l}\text { 3. Assure generalizations to } \\
\text { the natural environment }\end{array}$ & $\begin{array}{l}\text { Online consultations with the therapist, homework assignments; therapy tape review and analysis; } \\
\text { f. Structure the surroundings }\end{array}$ \\
$\begin{array}{l}\text { 5. Enhance practitioner capabilities } \\
\text { and motivation to treat }\end{array}$ & \begin{tabular}{l} 
Team work, supervision, consultation services \\
\hline
\end{tabular}
\end{tabular}

in the treatment of eating disorders but have not been evaluated as an augmentation strategy. According to Atkinson, under ideal facilitation, participants practicing mindfulness demonstrated significant improvement in weight and shape concerns, dietary restriction, thin-ideal internalization and psychosocial impairment relative to control by 6-month follow-up. Moreover, participants showed significant reductions in socio-cultural pressures [52].

Practicing mindful meditation when in recovery from an eating disorder or use in combination with naltrexone-bupropion can calm patients' mind and teach them to relax in environments that trigger destructive eating behaviors, thus helping them to make more sound decisions. Some articles listed the mindfulness-based eating awareness training (MB-EAT) program, consisting of 12 sessions, with a range of meditation practices, including breath awareness, mini-meditations, mindful eating meditations and exercises as an effective treatment approach [53]. During the course of the program, the incidence of binges, depression and eating issues all decreased significantly and remained lower during the 6-week follow-up period. Noticeably, the degree of improvement was in direct correlation to the time spent on the eating-related meditations. We believe that this treatment approach could be used in combination therapy of BED. However, it is unclear how to incorporate this time-consuming approach in the treatment of patients with inadequate response to naltrexone-bupropion.

Adolescent focused psychotherapy (AFP) is a form of individual psychotherapy from a self-psychology model. It helps patients to identify, tolerate and more effectively control their emotions. Initially, it is essential to build rapport between therapist and patient and develop a mutually understood conceptualization of abnormal eating behavior. The next phase includes gradual enhancement of individualization and independence from parents. Developing appropriate coping strategies to deal with the tasks of adolescence and engage in independent behaviors is a final stage. However, several RCTs indicated that AFP is less effective than family-based therapy (FBT) [54].

FBT is focused on weight restoration and aiming to empower parents and families to elicit change. FBT is an approach which externalizes the condition from the patient, empowering the family to work actively towards attenuating the disorder. This therapy could be used in combination with psychopharmacological modalities in order to help patients control their behavior. This is the only treatment that has well-established empirical evidence, but only in young patients with anorexia and bulimia. We believe that very similar approach could be used in treating patients with binge eating behavior or obesity. At least two RCTs have provided empirical evidence for FBT in bulimic and overweight adolescents. Unfortunately, no RCTs have been published examining the efficacy of treatment for adolescents with BED.

A study at Maudsley Hospital in 2012 focused on the use of FBT in the management of eating disorders among adolescents, including binge eating and bulimia nervosa. The primary focus is on modification of parental criticism, which may be higher in families of a patient with bulimia and binge eating than with AN, and on reduction of the shame and secrecy commonly surrounding binge eating and purging behaviors. This approach tends to be more collaborative in nature and can be useful in augmentation of specific pharmacotherapy.

In BED, this approach may include three phases: 1) Engage the entire family in the eating disorder management. In BED patients, the goal is to shift control of eating over to the parents. 2) Control over food decisions is gradually handed back to patient. 3) Help patient and his or her family navigate a return to normal trajectory of development.

Future research is needed to identify families for whom FBT does not work, determine adaptations to FBT that may increase its efficacy, develop ways to improve treatment adherence among clinicians and find ways to support caregivers better during treatment $[55,56]$.

Parental involvement is critical and central to this approach. However, it is possible that parents can get frustrated with refusal to eat, misinterpret refusal to eat and blame their child for bringing stress on the family. It has been mentioned that sometimes parents may escape from their role and become overly permissive. In order to be most effective, parents should be advised to align with one another and send clear messages regarding decisions about the child's meals and activity level. Additionally, young children may benefit from reminders to use rational responses to automatic thoughts. Age of young patients needs to be taken into consideration, and adaptations should be made for developmental level [55].

Sysko and Walsh carried out a study to determine the efficacy of self-help interventions for the treatment of BED as well as bulimia nervosa. The study involved the evaluation of 


\section{Aversion Therapy}

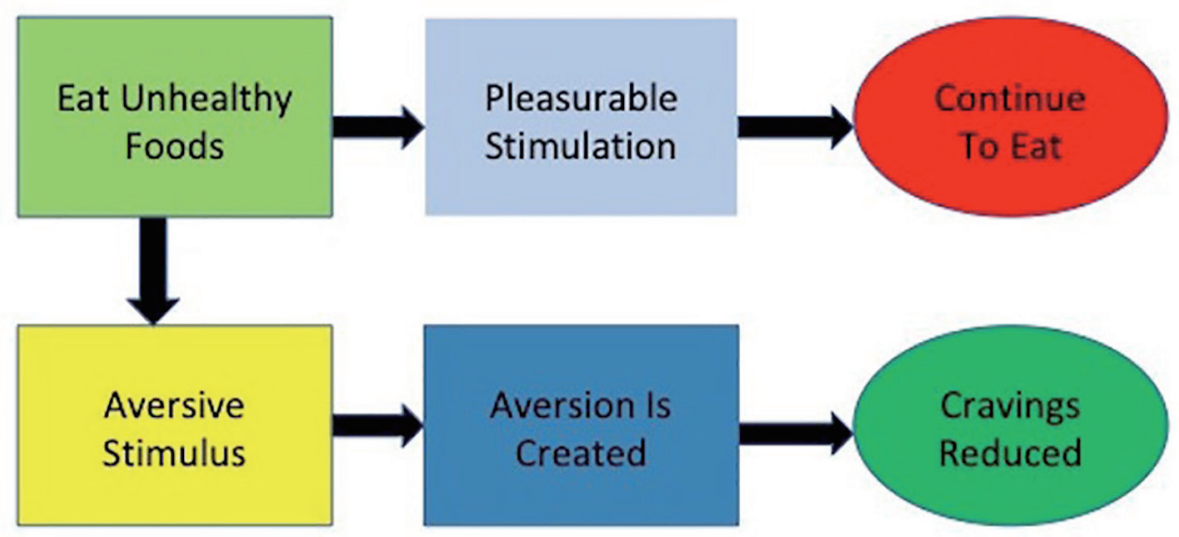

Figure 5. Aversion therapy of binge eating disorder.

the efficacy of self-help programs for the two eating disorders. A controlled study was employed. The study consisted of a waiting list for the control group. According to this study, selfhelp provided more benefits than the control waiting group. However, there is inadequate efficacy of this self-help treatment compared to others. This necessitates the requirement of further studies to be carried out on self-help types of treatment to determine the specific utility of this method in treatment of BED as well as bulimia nervosa [57].

Just like in the management of patients with opiate dependency, psychoeducation is very critical to achieve full clinical outcomes. In eating disorders, patients are given proper education with regard to weight and their eating habits. A lot of these patients have false beliefs towards their condition. A study carried out by Fairburn, proved that the characteristics among BED patients is highly associated with physical and social factors. The patients should be educated on all matters concerning their condition. In the study, patients were asked to read reliable information from eating disorder books. The information requested by their clinicians to read included, ineffectiveness of diuretics, laxatives and vomiting in management of heavy weight. Patients were also educated on body weight and interpretation of BMI. They were informed on the effects of treatments on their weight and the untoward effects of dieting and diet types that may induce the disease [58].

Drewnowski's research concluded that eating disorder patients associated calories with fat content to a greater extent than did controls and tended to dislike high-fat foods. In contrast, no differences in perceptions or preferences for carbohydrate foods were observed [59].

Aversion therapy is a very important treatment approach in managing both opiate withdrawal as well as eating disorders including BED. Aversion is a situation where an individual completely avoids the high urge to use something, for example excess food in BED. The taste of certain food can be made aversive through classical conditioning. Figure 5 provides additional details about aversion therapy.
According to research on aversion as a treatment option, it entails training of the brain to associate the behavior with a noxious stimulus. With this, there is behavioral modification focused on weaning the person from the bad habit. According to researchers, leaving unhealthy food at the store such that when the urge to eat comes, helps to resist the drive got eat by the inconvenience of traveling back to the store to get it [60]. However, we do not think that this therapy would be helpful in augmentation of psychopharmacological treatment of BED due to its aggressive approach and insufficient data about its effectiveness.

Exposure and response prevention (ERP) in the treatment of eating disorders is an uncommon strategy, but may be considered as an option in an augmentation-focused approach. Patients might be exposed to foods normally associated with overeating, but are prevented from excessive consumption of food. The goal is to create a hierarchy of feared food items and food situations each rated based upon the level of anxiety the stimulus provokes. Exposures can be experimental or more formalized and can be recorded to show habituation. Moreover, exposures can be imaginary, in person, with actual food, and with actual environments. Because the binge eating is not reinforced, this procedure eventually causes the response to extinguish. The goal is to complete $70 \%$ of hierarchy and be able to interact with food without engaging in BED behaviors. The binging behavior also decreases in frequency. Figure 6 describes this approach in bulimic patients. A similar approach can be possible to implement in binge eaters. Reilly et al proposed shifting to an inhibitory learning framework, considering treatment targets other than fear, and increasing variability in exposure techniques which represent three important areas for future study [61].

Group therapy is a very important treatment method in both eating disorders and in management of opiate dependence among patients. It is important to seek groups led by psychologists in the trial to wean oneself from poor eating habits. People get to meet with others struggling with the same condition. According to a study on the effectiveness of group therapy among 


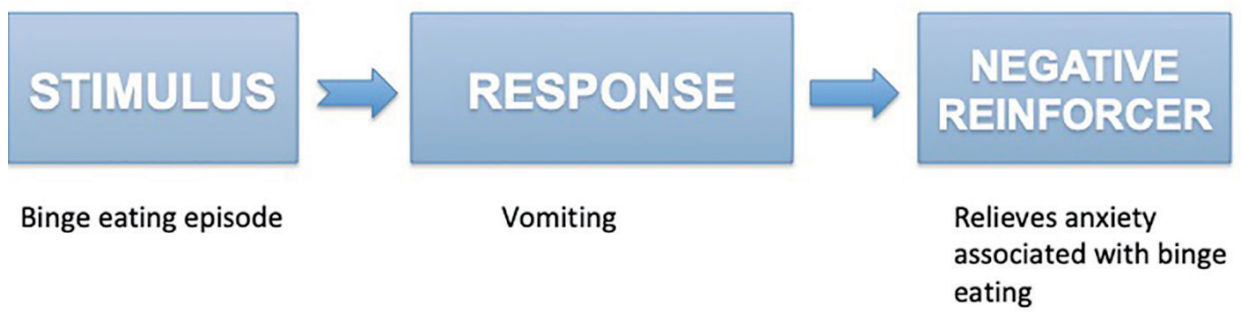

Figure 6. Exposure and response prevention strategy in the treatment of eating disorders.

substance abuse individuals, it was evident that the lives of people is basically shaped by the experiences of others in a group. An individual will become emotionally attached to other individuals in the group and the leader. In the groups the individuals get social support from others in times of trouble and pain. The groups enrich the individuals with guidance and support. The need for human contact is determined biologically. In the study, it was evident that group therapy is more beneficial than individual-based therapy. Several studies conducted among opiate addicts in methadone maintenance treatment (MMT) programs indicated that patients in the psychotherapy cohort showed less drug use than control subjects. This group difference was statistically significant at 6-month follow-up. These findings underscore the usefulness of group psychotherapy in eating disorder patients, especially in binge eaters on naltrexone-bupropion. Just like with substance abuse, binge patients improve from their conditions faster with group therapy. For example, Overeaters Anonymous is a 12-step program focused on abstinence from overeating, involving a sponsor (person of support to help with 12 steps and to contact when in high-risk situations). Patients on group therapy have better and faster clinical outcomes. Another possible alternative is participation in support groups that focus on providing insight through fellow members experiencing the same problems without using a 12 step-model [62] (Table $5[41-50,52-58,60])$.

\section{Challenges and Prognostic Factors}

Concerns about shape and weight are examples of the moderators and predictors of therapeutic efficacy of these interventions. Non-specific predictors of treatment efficacy include rapid response, pretreatment psychopathology, shape and weight concerns, and dietary restraint. Rapid response is defined as a greater than $65-70 \%$ decrease in binge eating by the fourth therapeutic session. Some studies propose that a $15 \%$ decrease in binge eating after CBT treatment was predictive of abstinence. Rapid responders are also more likely to show abstinence immediately after pharmacotherapeutic interventions than nonrapid responders. Pretreatment psychopathology issues such as a high psychopathology baseline and personality disorders predict higher post-treatment depression and binge eating, and decreased abstinence likelihood after treatment. With reference to concerns about body shape and weight, reduced body weight is fully predictive of abstinence post-CBT. Other studies show that clinical shape and weight overvaluation is the most pertinent abstinence predictor following fluoxetine therapy and
CBT. Lowering of rigid restraint and higher flexible dietary restraint over the course of therapy have been associated with better treatment outcomes. High negative affect coupled with the dietary restraint levels is predictive of treatment-resistant and more severe BED.

Low education levels may be associated with higher binge eating frequency and poorer outcomes post-IPT, behavioral weight loss and CBT. African Americans are more likely to quit treatment than Caucasians; however, Caucasians exhibit lesser global eating disorder reductions compared to their African American counterparts.

Earlier BED onset has also been associated with poorer post-treatment outcomes. There is an age-related difference in the associations based on the psychological interventions. For example, on fluoxetine therapy, younger participants had better outcomes compared to older individuals; this is conversely true for CBT since older participants were more likely to develop abstinence than the younger participants.

Other moderators predict treatment and include negative affect, shape and weight overvaluation and BED severity before treatment. In individuals receiving IPT, those with more compensatory and binge eating frequency, negative affect and shape and weight concerns showed the greatest abstinence. For CBT, those with lower compensatory and exciting tendencies, and a high binge eating frequency showed the highest abstinence. Better outcomes were found in individuals with greater previous binge frequency and lower self-esteem on CBT than fluoxetine.

Even when compliance has been achieved, there are still risks of relapse. Psychological interventions help patients identify cues when they are about to relapse. The cues identified include understanding and avoiding high-risk situations that could lead to dropping treatment and/or a binge eating episode. These techniques challenge the patient's expectations of perceived positive effects of the relapse, which helps patients make more informed choices in threatening situations.

\section{Limitations}

This article presents a general overview covering a specific topic, which provides primary information on this matter. We understand that this review may not provide the best available answers to focused questions.

The limitations of a literature review of this nature are the complete reliance on previously published research and the limited availability of these studies. We relied on a relatively limited 

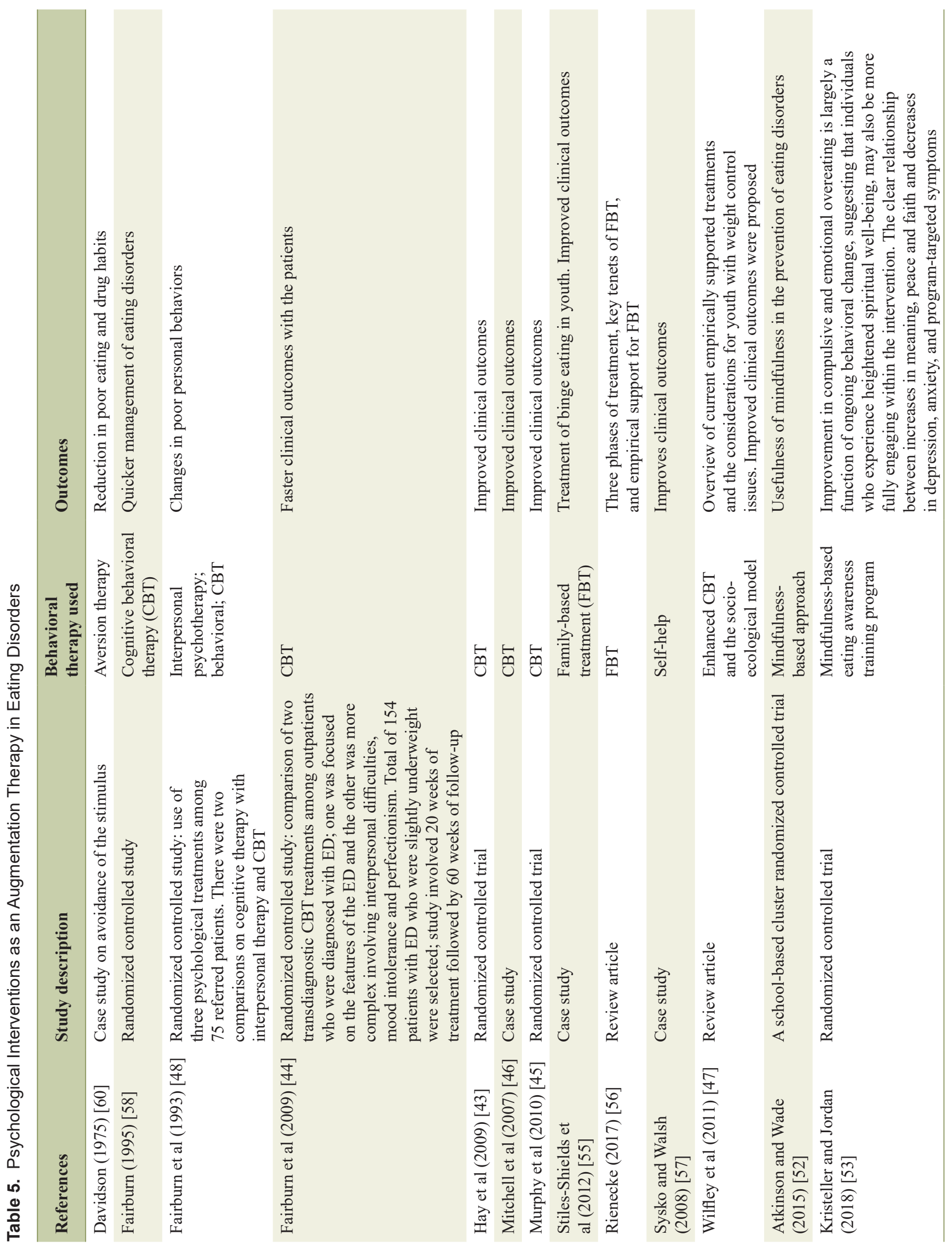


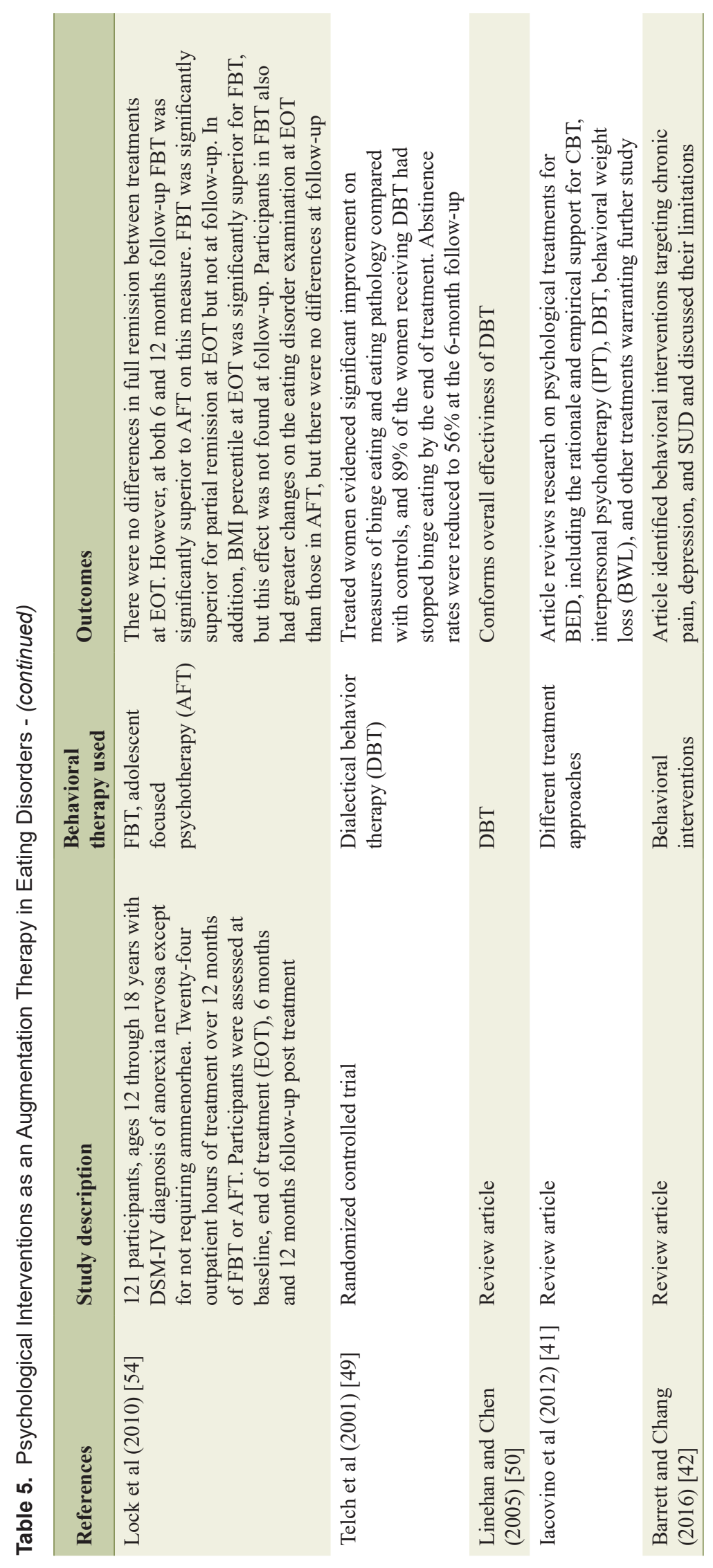


number of databases for the identification of potentially eligible articles and the selection of studies for inclusion in this review was frequently based on quality criteria. The assessment of study quality was often limited. This review has an accurate and up-todate bibliography that adheres to recognized style guidelines, but it does not compare psychological interventions to other techniques (e.g. pharmacological augmentation). It does not compare the effectiveness of each of the augmentation strategies. Moreover, it is only relevant for patients with severe eating disorders.

All studies include prescriptions to reduce caloric intake and lifestyle alteration recommendations. This limits the interpretation of the results with motivated patients and provides us with unclear information about how the preparation would work without diet/lifestyle alterations. For example, bupropion itself causes a much more significant loss of weight, which was determined in studies on the treatment of obesity compared to the analysis of depression studies, where loss of weight is not the main goal.

There are several factors to consider when reviewing research examining naltrexone-bupropion and other combinations [25]. First, the manufacturer of the combined pills sponsored many of these studies. Those studies were, however, perfectly conducted by experienced researchers. Second, these are all efficacy studies that were carried out under thorough experimental conditions, attracting carefully selected ideal patients. Efficacy studies that would demonstrate real preparation efficiency indicators were not conducted as of today. It is quite possible that study results may not reflect real results.

Third, most study reports were analyzed using the latest observation strategy. Loss of weight studies as a rule are characterized by the high screening rate; in the naltrexone-bupropion studies this indicator was higher than with about half of the subjects. Participants who left the group could maintain or not maintain the results registered at the time of screening.

Although these studies failed to find a correlation between food intake reduction and nausea, further studies will be necessary to clearly elucidate if this side effect might contribute to the naltrexone-induced suppression of food intake.

There are no gender-specific or sex hormone-sensitive interventions for BED. Recent studies explain the importance of genetic components on eating behavior, identifying a heritable effect of medium-high importance, and with a genderindependent transmission [63]. There is no exploratory study on specific factors that explain different gender responses to naltrexone in patients with BED. According to some studies, women are more likely to discontinue treatment with opiate antagonists when reporting severe pre-treatment psychiatric conditions, or nausea while in treatment [25]. It might be more difficult for patients with strong food cravings to successfully diet or lose weight. This may be especially relevant to women, especially during menstrual phases when they may be more sensitive to cravings and food cues. Surprisingly, weight cycling in women who diet was associated with increased frequency of binge eating behavior [64]. Additionally, recent meta-analyses of the few trials that do report gender differences found that women are less likely to succeed in treatment and female participants lose less weight than men [65].

Interestingly, testosterone is associated with lower risk of obesity and diabetes in men, but increased risk of obesity and diabetes in women. However, there is no reliable data about hormonal effects on binge eating behavior within and across sex and age, including increases in both hormones in men and women during adolescence and reductions in androgens in men and estrogens in women at older ages [66].

Due to a lack of data, there has been little research on age difference between naltrexone responders vs. naltrexone nonresponders. According to McElroy et al, BED is more common in male participants, has a later age of onset in adult cohorts and is more often associated with obesity than bulimia nervosa. Based on their data, the mean \pm standard deviation age of onset for BED was $25.4 \pm 1.2$ years as compared with 19.7 \pm 1.3 years for bulimia nervosa [67]. Although the evidence for naltrexone use in young patients (less than 18 years old) is lacking, the case series described naltrexone as well tolerated, and effective for the treatment of adolescents with binge eating and/or purging behavior [68]. Moreover, naltrexone/bupropion therapy carries a boxed warning common to the class regarding suicidal thoughts and behavior in children, adolescents and young adults age 24 and under [69]. No information is available on the relationship of age to the effects of naltrexone in geriatric patients, although changes in hepatic function tests have been described in obese elderly patients receiving naltrexone at doses higher than recommended (up to $300 \mathrm{mg}$ / day) for the treatment of alcoholism. Moreover, early studies on naltrexone for weight loss used high doses (e.g. $150 \mathrm{mg}$ and higher) and observed the elevation of liver transaminases. That is why naltrexone has to be prescribed with caution in cases of liver function impairment [70].

At the same time, we believe that the treatment dosage should not be the same for all patients with BED. For example, higher dosages may be administered in patients with morbid obesity, rapid drug metabolism, or specific genetic polymorphisms.

\section{Future Directions}

\section{Neurobiological perspective}

The importance of the endogenous opioid system modulating feeding behavior and other parameters that are crucial for the regulation of energy balance has been conclusively demonstrated by numerous preclinical and clinical reports. For instance, it seems clear that the complete lack of MOR and KOR causes important alterations in energy balance, particularly when mice are fed on fat-enriched diets. Moreover, the potential role of DOR deficiency has not been studied, and according to pharmacological data, it might be possible to find some important metabolic alterations after the disruption of DOR.

Although evidence suggests that behavioral effects of opioid antagonists have been demonstrated independently of side effects, further studies with specific genetic ablation of the different opioid receptors at neuronal clusters (nuclei) should be carried out in experimental animals in comparison with standard pharmacological approaches to ensure that the data generated can be interpreted correctly.

Of particular relevance is the location of opioid receptors in several areas of the mesolimbic dopamine system such as 
the VTA and the NAcc. It will be of great interest to generate and characterize mice lacking MOR, KOR or DOR in these specific brain areas in order to understand more precisely the molecular underpinnings modulating the actions of the endogenous opioid system on the hedonic properties of food. Also, the issue of gender should be taken into consideration since it is known that KOR agonists in males elicited greater suppression of food intake than in females.

Similarly, in humans mixed KOR/MOR ligands have been found to produce greater analgesia in women than in men. In contrast, in animals, selective KOR agonists have been found to produce greater antinociceptive effects in males than in females.

\section{Psychological perspective}

We believe that future directions in the management of BED include the development of cost-effective and efficient progressive care models, diversification in the testing of treatments in groups such as the youth and minorities, expansion of evidence-based practice in treatment, and treatment outcome improvement for non-responders. The propagation and implementation of scientific methods in practice will help identify the best training and treatment strategies across a wide range of clinical settings. We believe that the incorporation of knowledge about the individual variations in treatment response in treatment planning would assist in stepped-care strategy development. Consideration of subpopulation differences such as racial and ethnic differences in the adaptation of treatments would help in addressing BED issues in an acceptable and appropriate manner to the specific population. Moreover, further research on the brain reward system will improve the targeted behavioral and pharmacological therapies. The earlier identification of individuals at risk such as binge eating children is crucial since psychotherapeutic interventions are more effective at transitional and identity development periods such as adolescence.

\section{Conclusions}

There are still some important gaps in our knowledge surrounding several opioid-related issues. It should be also emphasized that, despite the large amount of data gleaned over the last few years regarding the involvement of opioid receptors as key players in food and alcohol reward, there are strong concerns as to which extent the outcome of studies on opiate antagonists can be interpreted as evidence for a direct role of opioids or whether these are consequences of the side effects associated with administration of these drugs. Moreover, the studies indicate the existence of marked gender and species differences in opioid receptor mediated biological effects. Future studies with a robust phenotype and genotype characterization are needed in order to better define and uncover patients that will benefit from treatment with drugs targeting the opioid system.

This report provides preliminary support for the use of psychotherapy as an adjunctive intervention to psychopharmacotherapy for BED. According to our understanding, the augmentation of naltrexone treatment with psychotherapy should be tried primarily on binge eating patients, in case naltrexone/ bupropion monotherapy is ineffective. Preliminary analysis of the literature showed a possible reduction in dysfunctional idiosyncratic thoughts as well as mild improvements in quality of life. However, these methods have never been used as an adjunctive strategy in patients on naltrexone. Psychotherapy in different forms could be a valuable addition to opiate antagonists by facilitating or enhancing cognitive changes; however, further studies are needed to confirm these promising ideas.

\section{Acknowledgments}

This work would not have been possible without the academic support of California Southern University. We are grateful to all of those with whom we have had the pleasure to work during this and other related projects. We thank our colleagues who provided insight and expertise that greatly assisted the research, although they may not agree with all of the interpretations/conclusions of this paper. We thank Dr. Heather Frederick, PhD, Faculty Mentor, School of Behavioral Sciences from California Southern University for comments that greatly improved the manuscript.

\section{Financial Disclosure}

We have no financial activities to disclose. We have no financial relationships (regardless of compensation amount) associated with the submitting author and all co-authors.

\section{Conflict of Interest}

We did not receive any payment or services from a third party (government, commercial, private foundation, etc.) for any aspect of the submitted work (such as grants, data monitoring board, study design, manuscript preparation, analysis, etc.). We do not have any patents, whether planned, pending or issued, that could be considered broadly relevant to this work. There are no interactions with any entity that could be considered broadly relevant to this work nor are there any other relationships/conditions/circumstances that present a potential conflict of interest.

\section{Author Contributions}

All authors of this research paper have directly participated in the planning, execution and analysis of this study. LV encouraged VZ to investigate an augmentation strategy and supervised the findings of this work. LV was in charge of overall direction and planning. LV developed the theoretical formalism, revised the manuscript, the main conceptual ideas and proof outline. VZ conceived the presented idea and carried out 
the literature review. VZ wrote the manuscript with support from LV. VZ aided in interpreting the results and worked on the manuscript. All authors of this paper have read and approved the final version submitted.

\section{Data Availability}

The authors declare that data supporting the findings of this study are available within the article.

\section{References}

1. Nogueiras R, Romero-Pico A, Vazquez MJ, Novelle MG, Lopez M, Dieguez C. The opioid system and food intake: homeostatic and hedonic mechanisms. Obes Facts. 2012;5(2):196-207.

2. Mendez IA, Ostlund SB, Maidment NT, Murphy NP. Involvement of endogenous enkephalins and beta-endorphin in feeding and diet-induced obesity. Neuropsychopharmacology. 2015;40(9):2103-2112.

3. Howick K, Griffin BT, Cryan JF, Schellekens H. From belly to brain: targeting the ghrelin receptor in appetite and food intake regulation. Int J Mol Sci. 2017;18(2):273.

4. St-Pierre DH, Rabasa-Lhoret R, Lavoie ME, Karelis AD, Strychar I, Doucet E, Coderre L. Fiber intake predicts ghrelin levels in overweight and obese postmenopausal women. Eur J Endocrinol. 2009;161(1):65-72.

5. St-Pierre DH, Karelis AD, Coderre L, Malita F, Fontaine J, Mignault D, Brochu M, et al. Association of acylated and nonacylated ghrelin with insulin sensitivity in overweight and obese postmenopausal women. J Clin Endocrinol Metab. 2007;92(1):264-269.

6. Hruby VJ, Cai M, Nyberg J, Muthu D. Approaches to the rational design of selective melanocortin receptor antagonists. Expert Opin Drug Discov. 2011;6(5):543-557.

7. Fetissov SO, Harro J, Jaanisk M, Jarv A, Podar I, Allik J, Nilsson I, et al. Autoantibodies against neuropeptides are associated with psychological traits in eating disorders. Proc Natl Acad Sci U S A. 2005;102(41):14865-14870.

8. Cottone P, Sabino V, Steardo L, Zorrilla EP. Opioid-dependent anticipatory negative contrast and binge-like eating in rats with limited access to highly preferred food. Neuropsychopharmacology. 2008;33(3):524-535.

9. Beck B. Neuropeptide $Y$ in normal eating and in genetic and dietary-induced obesity. Philos Trans R Soc Lond B Biol Sci. 2006;361(1471):1159-1185.

10. Tsujino N, Sakurai T. Role of orexin in modulating arousal, feeding, and motivation. Front Behav Neurosci. 2013;7:28.

11. Zheng H, Patterson LM, Berthoud HR. Orexin signaling in the ventral tegmental area is required for high-fat appetite induced by opioid stimulation of the nucleus accumbens. J Neurosci. 2007;27(41):11075-11082.

12. Levine AS, Grace MK, Cleary JP, Billington CJ. Naltrexone infusion inhibits the development of preference for a high-sucrose diet. Am J Physiol Regul Integr Comp
Physiol. 2002;283(5):R1149-1154.

13. Naleid AM, Grace MK, Cummings DE, Levine AS. Ghrelin induces feeding in the mesolimbic reward pathway between the ventral tegmental area and the nucleus accumbens. Peptides. 2005;26(11):2274-2279.

14. Levine AS, Grace M, Billington CJ, Zimmerman DM. Central administration of the opioid antagonist, LY255582, decreases short- and long-term food intake in rats. Brain Res. 1991;566(1-2):193-197.

15. Statnick MA, Tinsley FC, Eastwood BJ, Suter TM, Mitch $\mathrm{CH}$, Heiman ML. Peptides that regulate food intake: antagonism of opioid receptors reduces body fat in obese rats by decreasing food intake and stimulating lipid utilization. Am J Physiol Regul Integr Comp Physiol. 2003;284(6):R1399-1408.

16. Davis CA, Levitan RD, Reid C, Carter JC, Kaplan AS, Patte KA, King N, et al. Dopamine for "wanting" and opioids for "liking": a comparison of obese adults with and without binge eating. Obesity (Silver Spring). 2009; 17(6):1220-1225.

17. Bertino M, Beauchamp GK, Engelman K. Naltrexone, an opioid blocker, alters taste perception and nutrient intake in humans. Am J Physiol. 1991;261(1 Pt 2):R59-63.

18. Cota D, Tschop MH, Horvath TL, Levine AS. Cannabinoids, opioids and eating behavior: the molecular face of hedonism? Brain Res Rev. 2006;51(1):85-107.

19. Sinclair JD. Europe Patent No. EP 1681057 A1. 2006. Retrieved August 22, 2019, from https://patentimages. storage.googleapis.com/8c/15/41/c2637e66423d1a/ EP1681057A1.pdf.

20. Stolar MH. The effect of psychopharmacological agents on appetite and eating. In: Blinder BJ, Chaitin BF , Goldstein R (Ed). The Eating Disorders. PMA Publishing Corp. 1988:87-99.

21. Moore R, Mills IH, Forster A. Naloxone in the treatment of anorexia nervosa: effect on weight gain and lipolysis. J R Soc Med. 1981;74(2):129-131.

22. Blasio A, Steardo L, Sabino V, Cottone P. Opioid system in the medial prefrontal cortex mediates binge-like eating. Addict Biol. 2014;19(4):652-662.

23. Drewnowski A, Krahn DD, Demitrack MA, Nairn K, Gosnell BA. Naloxone, an opiate blocker, reduces the consumption of sweet high-fat foods in obese and lean female binge eaters. Am J Clin Nutr. 1995;61(6):12061212.

24. Holtzman SG. Suppression of appetitive behavior in the rat by naloxone: lack of effect of prior morphine dependence. Life Sci. 1979;24(3):219-226.

25. Billes SK, Sinnayah P, Cowley M. Naltrexone/bupropion for obesity: An investigational combination pharmacotherapy for weight loss. Pharmacol Res. 2014;84:1-11.

26. Giuliano C, Robbins TW, Nathan PJ, Bullmore ET, Everitt BJ. Inhibition of opioid transmission at the mu-opioid receptor prevents both food seeking and binge-like eating. Neuropsychopharmacology. 2012;37(12):2643-2652.

27. Lee MW, Fujioka K. Naltrexone for the treatment of obesity: review and update. Expert Opin Pharmacother. 2009;10(11):1841-1845.

28. Malcolm R, O'Neil PM, Sexauer JD, Riddle FE, Currey 
HS, Counts C. A controlled trial of naltrexone in obese humans. Int J Obes. 1985;9(5):347-353.

29. Kumar RB, Aronne L. Pharmacologic Treatment of Obesity. In A. B. Feingold KR, Endotext [Internet]. 2017. Retrieved from https://www.ncbi.nlm.nih.gov/books/NBK279038.

30. Selleck RA, Baldo BA. Feeding-modulatory effects of mu-opioids in the medial prefrontal cortex: a review of recent findings and comparison to opioid actions in the nucleus accumbens. Psychopharmacology (Berl). 2017;234(9-10):1439-1449.

31. Yeomans MR, Gray RW. Selective effects of naltrexone on food pleasantness and intake. Physiol Behav. 1996;60(2):439-446.

32. Yanovski SZ, Yanovski JA. Naltrexone extended-release plus bupropion extended-release for treatment of obesity. JAMA. 2015;313(12):1213-1214.

33. Gordon RJ, Panigrahi SK, Meece K, Atalayer D, Smiley $\mathrm{R}$, Wardlaw SL. Effects of opioid antagonism on cerebrospinal fluid melanocortin peptides and cortisol levels in humans. J Endocr Soc. 2017;1(10):1235-1246.

34. Billes SK, Sinnayah P, Cowley MA. Naltrexone/bupropion for obesity: an investigational combination pharmacotherapy for weight loss. Pharmacol Res. 2014;84:1-11.

35. McElroy SL, Guerdjikova AI, Kim DD, Burns C, HarrisCollazo R, Landbloom R, Dunayevich E. Naltrexone/ Bupropion combination therapy in overweight or obese patients with major depressive disorder: results of a pilot study. Prim Care Companion CNS Disord. 2013;15(3).

36. Rebello CJ, Greenway FL. Reward-induced eating: therapeutic approaches to addressing food cravings. Adv Ther. 2016;33(11):1853-1866.

37. Mason AE, Laraia B, Daubenmier J, Hecht FM, Lustig RH, Puterman E, Adler N, et al. Putting the brakes on the "drive to eat": Pilot effects of naltrexone and rewardbased eating on food cravings among obese women. Eat Behav. 2015;19:53-56.

38. Alger SA, Schwalberg MJ, Bigaouette JM, Howard LJ, Reid LD. Using drugs to manage binge-eating among obese and normal weight patients. In: Reid LD (Eds) Opioids, bulimia, and alcohol abuse and alcoholism. New York: Springer-Verlag; 1991:131-142.

39. Fairburn CG, Bailey-Straebler S, Basden S, Doll HA, Jones R, Murphy R, O'Connor ME, et al. A transdiagnostic comparison of enhanced cognitive behaviour therapy (CBT-E) and interpersonal psychotherapy in the treatment of eating disorders. Behav Res Ther. 2015;70:64-71.

40. Krupitsky E, Zvartau E, Woody G. Use of naltrexone to treat opioid addiction in a country in which methadone and buprenorphine are not available. Curr Psychiatry Rep. 2010;12(5):448-453.

41. Iacovino JM, Gredysa DM, Altman M, Wilfley DE. Psychological treatments for binge eating disorder. Curr Psychiatry Rep. 2012;14(4):432-446.

42. Barrett K, Chang YP. Behavioral interventions targeting chronic pain, depression, and substance use disorder in primary care. J Nurs Scholarsh. 2016;48(4):345-353.

43. Hay PP, Bacaltchuk J, Stefano S, Kashyap P. Psychological treatments for bulimia nervosa and binging. Cochrane Database Syst Rev. 2009;4:CD000562.
44. Fairburn CG, Cooper Z, Doll HA, O'Connor ME, Bohn K, Hawker DM, Wales JA, et al. Transdiagnostic cognitive-behavioral therapy for patients with eating disorders: a two-site trial with 60-week follow-up. Am J Psychiatry. 2009;166(3):311-319.

45. Murphy R, Straebler S, Cooper Z, Fairburn CG. Cognitive behavioral therapy for eating disorders. Psychiatr Clin North Am. 2010;33(3):611-627.

46. Mitchell JE, King WC, Pories W, Wolfe B, Flum DR, Spaniolas K, Bessler M, et al. Binge eating disorder and medical comorbidities in bariatric surgery candidates. Int J Eat Disord. 2015;48(5):471-476.

47. Wilfley DE, Kolko RP, Kass AE. Cognitive-behavioral therapy for weight management and eating disorders in children and adolescents. Child Adolesc Psychiatr Clin N Am. 2011;20(2):271-285.

48. Fairburn CG, Jones R, Peveler RC, Hope RA, O'Connor M. Psychotherapy and bulimia nervosa. Longer-term effects of interpersonal psychotherapy, behavior therapy, and cognitive behavior therapy. Arch Gen Psychiatry. 1993;50(6):419-428.

49. Telch CF, Agras WS, Linehan MM. Dialectical behavior therapy for binge eating disorder. J Consult Clin Psychol. 2001;69(6):1061-1065.

50. Linehan MM, Chen EY. Dialectical behavior therapy for eating disorders. In Encyclopedia of cognitive behavior therapy. Springer, Boston, MA. 2005;168-171.

51. Wysocki T, Harris MA, Buckloh LM, Mertlich D, Lochrie AS, Taylor A, Sadler M, et al. Effects of behavioral family systems therapy for diabetes on adolescents' family relationships, treatment adherence, and metabolic control. J Pediatr Psychol. 2006;31(9):928-938.

52. Atkinson MJ, Wade TD. Mindfulness-based prevention for eating disorders: A school-based cluster randomized controlled study. Int J Eat Disord. 2015;48(7):1024-1037.

53. Kristeller JL, Jordan KD. Mindful Eating: Connecting With the Wise Self, the Spiritual Self. Front Psychol. 2018;9:1271.

54. Lock J, Le Grange D, Agras WS, Moye A, Bryson SW, Jo B. Randomized clinical trial comparing family-based treatment with adolescent-focused individual therapy for adolescents with anorexia nervosa. Arch Gen Psychiatry. 2010;67(10):1025-1032.

55. Stiles-Shields C, Hoste RR, Doyle PM, Le Grange D. A review of family-based treatment for adolescents with eating disorders. Rev Recent Clin Trials. 2012;7(2):133140.

56. Rienecke RD. Family-based treatment of eating disorders in adolescents: current insights. Adolesc Health Med Ther. 2017;8:69-79.

57. Sysko R, Walsh BT. A critical evaluation of the efficacy of self-help interventions for the treatment of bulimia nervosa and binge-eating disorder. Int J Eat Disord. 2008;41(2):97-112.

58. Fairburn CG. Overcoming binge eating. Overcoming Binge Eating. 1995. Retrieved from https://www.cabdirect.org/cabdirect/abstract/19961409936.

59. Drewnowski A, Pierce B, Halmi KA. Fat aversion in eating disorders. Appetite. 1988;10(2):119-131. 
60. Davidson AB. Obesity: two behavioral approaches to weight reduction. Dissertations and Theses. 1975:2218.

61. Reilly EE, Anderson LM, Gorrell S, Schaumberg K, Anderson DA. Expanding exposure-based interventions for eating disorders. Int J Eat Disord. 2017;50(10):11371141.

62. Center for substance abuse treatment. Substance abuse treatment: group therapy. Rockville (MD): Substance Abuse and Mental Health Services Administration (US); 2005. (Treatment Improvement Protocol (TIP) Series, No. 41.) 1 Groups and Substance Abuse Treatment. Available from: https://www.ncbi.nlm.nih.gov/books/NBK64223/.

63. Thornton LM, Mazzeo SE, Bulik CM. The heritability of eating disorders: methods and current findings. Curr Top Behav Neurosci. 2011;6:141-156.

64. Field AE, Manson JE, Taylor CB, Willett WC, Colditz GA. Association of weight change, weight control practices, and weight cycling among women in the Nurses' Health Study II. Int J Obes Relat Metab Disord. 2004;28(9):1134-1142.

65. Robertson C, Avenell A, Boachie C, Stewart F, Archibald D, Douglas F, Hoddinott P, et al. Should weight loss and maintenance programmes be designed differently for men? A systematic review of long-term randomised controlled trials presenting data for men and women: The ROMEO project. Obes Res Clin Pract. 2016;10(1):70-84.

66. Gray A, Feldman HA, McKinlay JB, Longcope C. Age, disease, and changing sex hormone levels in middle-aged men: results of the Massachusetts Male Aging Study. J Clin Endocrinol Metab. 1991;73(5):1016-1025.

67. McElroy SL, Guerdjikova AI, Mori N, O'Melia AM. Pharmacological management of binge eating disorder: current and emerging treatment options. Ther Clin Risk Manag. 2012;8:219-241.

68. Stancil SL, Adelman W, Dietz A, Abdel-Rahman S. Naltrexone reduces binge eating and purging in adolescents in an eating disorder program. J Child Adolesc Psychopharmacol. 2019;29(9):721-724.

69. Guerdjikova AI, Walsh B, Shan K, Halseth AE, Dunayevich E, McElroy SL. Concurrent improvement in both binge eating and depressive symptoms with naltrexone/bupropion therapy in overweight or obese subjects with major depressive disorder in an open-label, uncontrolled study. Adv Ther. 2017;34(10):2307-2315.

70. Leroy A, Carton L, Gomajee H, Bordet R, Cottencin O. Naltrexone in the treatment of binge eating disorder in a patient with severe alcohol use disorder: a case report. Am J Drug Alcohol Abuse. 2017;43(5):618-620. 